ANNALES

POLONICI MATHEMATICI

XLV (1985)

\title{
Models for doubly commuting contractions
}

\author{
by Marek SŁocińskı (Cracow)
}

\begin{abstract}
The idea of Sz.-Nagy and Foias model for a contraction is generalized for the pair of doubly commuting contraction. The operators are described as backward shifts on vector valued $L^{2}$ and $H^{2}$ spaces on bidisc restricted to invariant subspace defined by some bounded analytic operator functions.
\end{abstract}

In what follows $H$ is a separable Hilbert space with inner product $(x, y)$; $x, y \in H$ and norm $\|x\|=\sqrt{(x, x)} ; x \in H \cdot L(H)$ stands for the algebra of all bounded linear operators on $H$. For $T \in L(H), T^{*}$ denotes the adjoint of $T$. The operator $T \in L(H)$ is called contraction if $\|T\| \leqslant 1$. Operators $T_{1}$ and $T_{2}$ doubly commute if $T_{1} T_{2}=T_{2} T_{1}$ and $T_{1}^{*} T_{2}=T_{2} T_{1}^{*}$. In the present paper we give, using the dilation theory, a functional model for the pair of doubly commuting contractions on a separable Hilbert space.

First we give the decomposition of the pair of doubly commuting contraction. Let $T_{1}$ and $T_{2}$ be a pair of doubly commuting contractions on the space $H$. If the decomposition $H=H_{1} \oplus H_{2}$ is the canonical decomposition of $T_{1}$ (see Theorem I.3, 2 of [1]) on the unitary and completely nonunitary parts, then $H_{1}=\bigcap_{n \neq 0} \operatorname{Ker} D_{T_{1}^{n}}$ where for $n \geqslant 0 \quad D_{T_{1}^{n}}=$ $\left(I-T_{1}^{* n} T_{1}^{n}\right)^{1 / 2}$ and for $n<0 D_{T_{1}^{*}}=\left(I-T_{1}^{|n|} T_{1}^{*|n|}\right)^{1 / 2}$. Since $T_{1}$ and $T_{2}$ doubly commute, $T_{2}$ and $T_{2}^{*}$ commute with $\left(I-T_{1}^{* n} T_{1}^{n}\right)$ and $\left(I-T_{1}^{|n|} T_{1}^{*|n|}\right)$ for every $n \geqslant 0$. Consequently $T_{2}$ and $T_{2}^{*}$ commute with $D_{T_{1}^{n}}$ for every $n \neq 0$. If $x \in \operatorname{Ker} D_{T_{1}^{n}}$ then $D_{T_{1}^{n}} T_{2} x=T_{2} D_{T_{1}^{n}} x=0$, hence $T_{2} x \in \operatorname{Ker} D_{T_{1}^{n}}$. Analogically $T_{2}^{*} x \in \operatorname{Ker} D_{T_{1}^{n}}$ for $x \in \operatorname{Ker} D_{T_{1}^{n}}$. This implies that, for every $n \neq 0$, Ker $D_{T_{1}^{n}}$ is reducing subspace for $T_{2}$ and consequently $H_{1}$ reduces $T_{2}$. The same consideration applied to the pairs $T_{1 \mid H_{1}}, T_{2 \mid H_{1}}$ and $T_{1 \mid H_{2}}, T_{2 \mid H_{2}}$, which evidently are doubly commutative, proves the following:

Proposition 1. Suppose that $T_{1}$ and $T_{2}$ are doubly commuting contractions on the space. Then there is a decompostion $H=\bigoplus_{i=1}^{4} H_{i}$ such that $H_{i}$ reduces $T_{1}$ and $T_{2}(i=1, \ldots, 4)$ and the following conditions hold:

$T_{1 \mid H_{1}}$ and $T_{2 \mid \mathrm{H}_{1}}$ are unitary operators, 
$T_{1 \mid H_{2}}$ is completely nonunitary and $T_{2 \mid H_{2}}$ is unitary,

$T_{1 \mid H_{3}}$ is unitary and $T_{2 \mid H_{3}}$ is completely nonunitary,

$T_{1 \mid H_{4}}$ and $T_{2 \mid H_{4}}$ are completely nonunitary.

Now it is easy to see that to find the model of doubly commuting contractions it is sufficient to solve this problem in two cases:

(I) $T_{1}$ and $T_{2}$ are completely nonunitary.

(II) $T_{1}$ is completely nonunitary and $T_{2}$ is unitary.

We begin with case (I). The following result, which is the consequence of Theorem 2 of [2], will be useful in our work:

THEOREM 0. Suppose that $T_{1}$ and $T_{2}$ are doubly commuting contractions on the space $H$. Then there is the minimal unitary dilation $U_{1}, U_{2}$ of the pair $T_{1}, T_{2}$ such that

(0.1) the dilation space $K$ has the form $K=\underset{i, j=-\infty}{\bigoplus} H_{i, j}$ where

$$
\begin{aligned}
& H_{0,0}=H, \quad H_{1,0}=\overline{\left(U_{1}-T_{1}\right) H}, \quad H_{0,1}=\overline{\left(U_{2}-T_{2}\right) H}, \\
& H_{-1,0}=\overline{\left(U_{1}^{*}-T_{1}^{*}\right) H}, \quad H_{0,-1}=\overline{\left(U_{2}^{*}-T_{2}^{*}\right) H}, \\
& H_{1,1}=\overline{\left(U_{1} U_{2}-U_{1} T_{2}-U_{2} T_{1}+T_{1} T_{2}\right) H}, \\
& H_{1,-1}=\overline{\left(U_{1}^{*} U_{2}-U_{1} T_{2}^{*}-U_{2} T_{1}^{*}+T_{1} T_{2}^{*}\right) H}, \\
& H_{-1,1}=\overline{\left(U_{1}^{*} U_{2}-U_{1}^{*} T_{2}-U_{2} T_{1}^{*}+T_{1}^{*} T_{2}\right) H}, \\
& H_{-1,-1}=\overline{\left(U_{1}^{*} U_{2}^{*}-U_{1}^{*} T_{2}^{*}-U_{2}^{*} T_{1}^{*}+T_{1}^{*} T_{2}^{*}\right) H},
\end{aligned}
$$

and for $n, m \geqslant 1$

$$
\begin{gathered}
H_{n, 0}=U_{1}^{n-1} H_{1,0}, \quad H_{-n, 0}=U_{1}^{*(n-1)} H_{-1,0} ; \\
H_{0, m}=U_{2}^{m-1} H_{0,1}, \quad H_{0,-m}=U_{2}^{*(m-1)} H_{0,-1}, \\
H_{n, m}=U_{1}^{n-1} U_{2}^{m-1} H_{1,1} ; \quad H_{-n,-m}=U_{1}^{*(n-1)} U_{2}^{*(m-1)} H_{-1,-1}, \\
H_{n,-m}=U_{1}^{n-1} U_{2}^{*(m-1)} H_{1,-1} ; \quad H_{-n, m}=U_{1}^{*(n-1)} U_{2}^{m-1} H_{-1,1}, \\
U_{1} H_{-1, j} \oplus H_{0, j}=H_{0, j} \oplus H_{1, j}, \quad U_{2} H_{j,-1} \oplus H_{j, 0}=H_{j, 0} \oplus H_{j, 1}
\end{gathered}
$$

for every integer number $j$.

Now, we can define the following spaces:

$$
\begin{array}{lll}
H_{. j}=\bigoplus_{i=-\infty}^{x} H_{i, j}, & H_{. j}^{+}=\bigoplus_{i=0}^{\infty} H_{i, j} & (j=0,1,2, \ldots), \\
H_{i .}=\bigoplus_{j=-x}^{\infty} H_{i, j}, & H_{i .}^{+}=\bigoplus_{j=0}^{\infty} H_{i, j} & (i=0,1,2, \ldots),
\end{array}
$$


and

$$
K_{1}^{+}=\bigoplus_{i=0}^{x} H_{i .}=\bigoplus_{j=-\infty}^{x} H_{. j}^{+}, \quad K_{2}^{+}=\bigoplus_{j=0}^{x} H_{. j}=\bigoplus_{i=-\infty}^{x} H_{i}^{+}
$$

$$
K^{+}=\bigoplus_{i, j=0}^{\infty} H_{i, j}=\bigoplus_{i=0}^{\infty} H_{i .}^{+}=\bigoplus_{j=0}^{\infty} H_{\cdot j}^{+}
$$

A subspace $L \subset H$ is called a wandering subspace for the unitary operator $U$ on $H$ if $U^{n} L \perp L$ for every $n \neq 0$. $L$ is called wandering for the pair of unitary operators $U_{1}, U_{2}$ on $H$ if $U_{1}^{n} U_{2}^{m} L \perp L$ for every $n, m$ such that $|m|+|n|$ $>0$. If $L$ is wandering subspace for $U_{i}$, then we can define the spaces $M_{i}(L)$ and $M_{i}^{+}(L)$ as follows $M_{i}(L)=\bigoplus_{n=-\infty}^{\infty} U_{i}^{n} L$ and $M_{i}^{+}(L)=\bigoplus_{n=0}^{x} U_{i}^{n} L$. If $L$ is wandering for the pair $U_{1}, U_{2}$, then $M(L)$ and $M^{+}(L)$ denote the spaces $M(L)=\bigoplus_{n, m=-\infty}^{\infty} U_{1}^{n} U_{2}^{m} L$ and $M^{+}(L)=\bigoplus_{n, m=0}^{\infty} U_{1}^{n} U_{2}^{m} L$.

Now we prove the following:

Lemma 1. Suppose that $T_{1}$ and $T_{2}$ are doubly commuting contractions on $H$. Let $U_{1}, U_{2}$ be a unitary dilation of $T_{1}, T_{2}$ as in Theorem 0 . Then with notations as above the following conditions hold true:

(1.1) the spaces $H_{-1,-1}, H_{-1,1}, H_{1,-1}, H_{1,1}$ are wandering subspaces for the pair $U_{1}, U_{2}$,

(1.2) $H_{0,-1}, H_{0,1}$ and $H_{-1,0}, H_{1,0}$ are wandering subspaces for $U_{2}$ and $U_{1}$ respectively,

(1.3) $H_{. i}^{+}$and $H_{i .}^{+}$are invariant subspaces for $U_{1}$ and $U_{2}$ respectively ( $i$ $=0, \pm 1, \pm 2, \ldots)$,

(1.4) $H_{. i}$ and $H_{i .}$ reduce $U_{1}$ and $U_{2}$ respectively $(i=0, \pm 1, \pm 2, \ldots)$.

Proof. Theorem 0 implies that for $n, m \geqslant 0, n+m>0$ we have $U_{1}^{n} U_{2}^{m} H_{1,1}=H_{1+m, 1+m} \perp H_{1,1}$ and consequently $U_{1}^{n} U_{2}^{m} H_{1,1} \perp H_{1,1}$ for $n, m \leqslant 0$ and $|n|+|m|>0$. If $n \geqslant 0, m \leqslant 0$ and $n+|m|>0$ and $x, y \in H_{1,1}$, then $U_{1}^{n} x \in H_{1+n, 1}$ and $U_{2}^{-m} y=U_{2}^{|m|} y \in H_{1,|m|+1}$, hence $\left(U_{1}^{n} U_{2}^{m} x, y\right)$ $=\left(U_{1}^{n} x, U_{2}^{-m} y\right)=0$. Thus $U_{1}^{n} U_{2}^{m} H_{1,1} \perp H_{1,1}$ for $n \geqslant 0, m<0$ and $n+$ $+|m|>0$. Consequently $U_{1}^{n} U_{2}^{m} H_{1,1} \perp H_{1,1}$ for $n \leqslant 0, m \geqslant 0$ and $|n|+m>0$. So we have proved that $H_{1,1}$ is the wandering subspace for the pair $U_{1}, U_{2}$. The rest of (1.1) we prove analogically.

Since for $n \geqslant 0, U_{2}^{n} H_{0,1}=H_{0, n+1}, H_{0,1}$ is a wandering subspace for $U_{2}$. This gives (1.2).

Now we shall show that $H_{d i}^{+}$is invariant for $U_{1}$. It is sufficient to show that for $j \geqslant 0, U_{1} H_{j, i} \subset H_{d i}^{+}$. If $j>0$ then $U_{1} H_{j, i}=H_{j+1, i} \subset H_{. i}^{+}$. For $j=0$, by (0.2) we have $U_{1} H_{0, i} \subset H_{0, i} \oplus H_{1, i} \subset H_{d}^{+}$which completes the proof of (1.3). 
To prove (1.4) it is sufficient to show that for every $j, U_{1} H_{j, i} \subset H_{d}$ and $U_{1}^{*} H_{j, i} \subset H_{j}$. If $j \geqslant 1$ then $U_{1} H_{j, i}=H_{j+1, i} \subset H_{j}$. If $j=0,-1$ then by $(0.2)$ we get $U_{1} H_{j, i} \subset H_{0, i} \oplus H_{1, i} \subset H_{, i}$. For $j \leqslant-1$ we have $U_{1} H_{j, i}$ $=H_{j+1, t} \subset H_{d}$. Thus $U_{1} H_{d} \subset H_{. i}$. It is easy to see that (0.2) implies that $U_{1}^{*} H_{1, i} \oplus H_{0, i}=H_{0, i} \oplus H_{-1, i}$. By using this, similar proof as above shows that $U_{i}^{*} H_{. i} \subset H_{. i}$, which completes the proof of our lemma.

If $L$ is a subspace of the Hilbert space $K$ we denote by $P_{L}$ the orthogonal projection of $K$ onto $L$ : Our second result will be the following:

Lemma 2. Suppose that $T_{1}$ and $T_{2}$ are doubly commuting contractions on $H$. Let $U_{1}$ and $U_{2}$ be a unitary dilation of $T_{1}, T_{2}$ as in Theorem 0. If $T_{1, i}=P_{H_{0, i}} U_{1 \mid H_{0, i}}$ and $T_{2, i}=P_{H_{i, 0}} U_{2 \mid H_{i, 0}}, P_{1, i}$ is the orthogonal projection of $H_{i}$ onto $M_{1}\left(H_{-1, i}\right)$ and $P_{2, i}$ is the orthogonal projection of $H_{i .}$ onto $M_{2}\left(H_{i,-1}\right)(i=0, \pm 1, \pm 2, \ldots)$, then the following conditions hold true:

(2.1) $U_{1 \mid H_{i j}}$ and $U_{2 \mid H_{i .}}$ are minimal unitary dilations of $T_{1, i}$ and $T_{2, i}$ respectively $(i=0, \pm 1, \pm 2, \ldots)$,

(2.2) if $T_{i}$ is completely nonunitary, then $T_{i, j}$ is completely nonunitary $(i=1,2, j=0, \pm 1, \pm 2, \ldots)$,

(2.3) if for the contraction $T$ on $H$ and its minimal unitary dilation $U, L_{T}^{+}$ and $L_{T}^{-}$denote the spaces $L_{T}^{+}=\overline{(U-T) H}$ and $L_{T}^{-}=\overline{\left(U^{*}-T^{*}\right) H}$, then $\quad L_{T_{1, i}}^{+}=H_{1, i}, \quad L_{T_{1, i}}^{-}=H_{-1, i}, \quad L_{T_{2, i}}^{+}=H_{i, 1}, \quad L_{T_{2, i}}^{-}=H_{i,-1} \quad(i$ $=0, \pm 1, \pm 2, \ldots)$.

Proof. If $x \in H_{0, i}$ then by (0.2) we get that $U_{1} x=x_{0}+x_{1}$ where $x_{k} \in H_{k, i}$. Thus $T_{1, i} x=P_{H_{0, i}} U_{1} x=x_{0}$. If follows that $U_{1} x=T_{1, i} x+x_{1}$ where $x_{1} \perp H_{0, i}$. Using mathematical induction one can prove that $U_{1}^{n} x$ $=T_{1, i}^{n} x+x_{1}$ where $x_{1} \perp H_{0, i}$. This implies that $U_{1 \mid H_{d i}}$ is a unitary dilation of $T_{1, i}$. To finish the proof of (2.1) we have to show that $H_{a i}=\bigvee_{n=-\infty}^{\infty} U_{1}^{n} H_{0, i}$. Since $H_{0, i} \subset H_{j}$, by (1.4) we get $\bigvee_{n=-\infty}^{\infty} U_{1}^{n} H_{0, i} \subset H_{, i}$. Suppose now, that $y \in H_{i}$ and $y \perp \bigvee_{n=-\infty}^{\infty} U_{1}^{n} H_{0, i}$. Since $K=\bigvee_{n, m=-\infty}^{\infty} U_{1}^{n} U_{2}^{m} H$, we are to show that $\left(U_{1}^{n} U_{2}^{m} x, y\right)=0$ for every integer numbers $n, m$ and $x \in H=H_{0,0}$. Fix $n, m$ and $x$. It is easy to see, by (0.2) and definition of $H_{0, j}$ that $U_{2}^{m} x=\sum_{j=0}^{m} x_{j}$ where $x_{j} \in H_{0, j}$. Since $x_{j} \in H_{0 j}$, by (1.4) we get that $U_{1}^{n} x_{j} \in H_{. j}$ which for $j \neq i$ is orthogonal to $H_{d} \supset H_{0, i}$. Consequently, for $j \neq i$, we have $\left(U_{1}^{n} x_{j}, y\right)=0$. If $j=i$ then $\left(U_{1}^{n} x_{i}, y\right)=0$, by our assumption that $y \perp \bigvee_{n=-\infty}^{\infty} U_{1}^{n} H_{0, i}$. Finally $\left(U_{1}^{n} U_{2}^{m} x, y\right)=\sum_{j=0}^{m}\left(U_{1}^{n} x_{j}, y\right)=0$ which completes the proof of $(2.1)$. 
Suppose now, that $x \in H_{0, i}$ has the following property: $\left\|T_{1, i}^{n} x\right\|$ $=\left\|T_{1, i}^{* n} x\right\|=\|x\|$ for every $n \geqslant 0$. To prove (2.2) it is sufficient to show that $x=0$ if $T_{1}$ is completely nonunitary. For $i=0$ this is obvious because $T_{1,0}=T_{1}$. Consider now the case $i>0$. If $j=-i$ then by $(0.2)$ we infer that $U_{2}^{j} x=x_{0}+x_{1}$ where $x_{k} \in H_{0, k}$. Since $H_{0}$. reduces $U_{2}$ (see (1.4)) we have the following equalities:

$$
\begin{aligned}
\left\|T_{1, i}^{n} x\right\|^{2} & =\left\|U_{2}^{-i} T_{1, i}^{n} x\right\|^{2} \\
& =\left\|U_{2}^{-i} P_{H_{0, i}} U_{1}^{n} x\right\|^{2}=\left\|P_{H_{0} .} U_{2}^{-i} U_{1}^{n} x\right\|^{2}=\left\|P_{H_{0} .} U_{1}^{n} x_{0}+P_{H_{0} .} U_{1}^{n} x_{1}\right\|^{2} \\
& =\left\|P_{H_{0,0}} U_{1}^{n} x_{0}+P_{H_{0,1}} U_{1}^{n} x_{1}\right\|^{2}=\left\|T_{1,0}^{n} x_{0}\right\|^{2}+\left\|T_{1,1}^{n} x_{1}\right\|^{2} .
\end{aligned}
$$

In fact, to prove the third and the sixth equality it is sufficient to show that $\boldsymbol{P}_{\boldsymbol{H}_{0 .}} y=\boldsymbol{P}_{\boldsymbol{H}_{0, i}} y$ for $y \in H_{s i}$. Let $y \in H_{i}$. Then $y=\sum_{j=-\infty}^{\infty} y_{j}$ where $y_{j} \in H_{j, i}$. If $j \neq 0$ then $H_{j, i} \perp H_{0, k}$ for every $k$, hence $H_{j, i} \perp H_{0}$. It follows that if $j \neq 0$ then $P_{H_{0}} y_{j}=0$. If $j=0$ then $P_{H_{0}} y_{0}=y_{0}$ because $y_{0} \in H_{0, i} \subset H_{0 .}$. Consequently $P_{H_{0 .}} y=y_{0}$. On the other hand, if $j \neq 0$ then $H_{j, i} \perp H_{0, i}$, hence $P_{H_{0, i}} y_{j}=0$. For $j=0$ we have $P_{H_{0, i}} y_{0}=y_{0}$. Finally we have $P_{H_{0, i}} y=y_{0}$ which proves that $P_{H_{0},} y=P_{H_{0, j}} y$. So we have proved that $\left\|T_{1, i}^{n} x\right\|^{2}=\left\|T_{1,0}^{n} x_{0}\right\|^{2}+$ $+\left\|T_{1,1}^{n} x_{1}\right\|^{2}$. On the other hand, $\|x\|^{2}=\left\|U_{2}^{-i} x\right\|^{2}=\left\|x_{0}\right\|^{2}+\left\|x_{1}\right\|^{2}$. Our assumption about $x$ implies that $\left\|x_{0}\right\|^{2}+\left\|x_{1}\right\|^{2}=\left\|T_{1}^{n} x_{0}\right\|^{2}+\left\|T_{1,1}^{n} x_{1}\right\|^{2}$ $\leqslant\left\|T_{1}^{n} x_{0}\right\|^{2}+\left\|x_{1}\right\|^{2}$. Consequently $\left\|x_{0}\right\|^{2} \leqslant\left\|T_{1}^{n} x_{0}\right\|^{2} \leqslant\left\|x_{0}\right\|^{2}$ which proves that $\left\|T_{1}^{n} x_{0}\right\|=\left\|x_{0}\right\|$ for every $n>0$. Analogically we prove that $\left\|T_{1}^{n} x_{0}\right\|$ $=\left\|x_{0}\right\|$ for every $n>0$. Since $T_{1}$ is completely nonunitary, these equalities imply that $x_{0}=0$. Consequently $U_{2}^{-i} x=x_{1} \in H_{1,0} \perp H_{0,0}$. For $j \leqslant-i$ we have $U_{2}^{j} x=U_{2}^{j+i} U_{2}^{-1} x \subset U_{2}^{j+i} H_{0,1}=H_{0,1+j+i} \perp H_{0,0}$. We have proved that $U_{2}^{j} x H_{0,0}$ for every $j$. Consequently $x \perp \bigvee_{n=-x}^{\infty} U_{2}^{n} H_{0,0}$. Now, (2.1) implies that $x \perp H_{0}$. But $x \in H_{0 .}$, hence $x=0$ which ends the proof of (2.2) for $i<0$. For $i>0$, the proof is similar.

Now we shall prove that $\overline{\left(U_{1}-T_{1, i}\right) H_{0, i}}=H_{1, i}$. If $x \in H_{0, i}$ then $U_{1} x$ $=T_{1, i} x+x_{1}$ where $x_{1} \in H_{1, i}$ (see the proof of (2.1)). It follows that $\left(U_{1}-\right.$ $\left.-T_{1, i}\right) x=x_{1} \in H_{1, i}$, hence $L_{T_{1, i}}^{+} \subset H_{1, i}$. Suppose now, that $y \in H_{1, i}$ and $y \perp L_{T_{1}, i}^{+}$. Now, if $x \in H_{0, i}$ then $0=\left(y ;\left(U_{1}-T_{1, i}\right) x\right)=\left(y, U_{1} x\right)-\left(y, T_{1, i} x\right)$ $=\left(y, U_{1} x\right)$. The last equality holds because $T_{1, i} x \in H_{0, i}$ and $y \in H_{1, i}$. Let $x \in H_{0, i}$. Then it is easy to see that for $n>1, U_{1}^{n-1} x=x_{0}+x_{1}$ where $x_{1} \in H_{0, i}$ and $x_{1} \in \bigoplus_{j=1}^{\infty} H_{j, i}$. It follows that for $n>1, U_{1}^{n} x=U_{1} x_{0}+U_{1} x_{1}$ where $x_{0} \in H_{0, i}$ and $U_{1} x_{1} \in \bigoplus_{j=2}^{\infty} H_{j, i} H_{1, i}$. Consequently $\left(y, U_{1}^{n} x\right)=\left(y, U_{1} x_{0}\right)+$ $+\left(y, U_{1} x_{1}\right)=0$ for every $n>1$. Since for $n \geqslant 0, H_{0, i} \perp H_{1+n, i}=U_{1}^{n} H_{1, i}$, we have $U_{1}^{-n} H_{0, i} \perp H_{1, i}$ for every $n \geqslant 0$. This implies that for $n \leqslant 0,\left(y, U_{1}^{n} x\right)$ 
$=0$. We have proved that for every integer number $n$ and every $x \in H_{0, i}$, $\left(y, U_{1}^{n} x\right)=0$, hence $y \perp \bigvee_{n=-\infty}^{\infty} U_{1}^{n} H_{0, i}$. It follows by (2.1) that $y \in H_{-i}$. But $y \in H_{j}$, so $y=0$. This proves that $L_{T_{1, i}}^{+}=H_{1, i}$. The rest of (2.3) we prove analogically, which completes the proof of Lemma 2.

Now, applying Theorems I.1.4 and I.2.1 of [1] and Lemma 2 to the operators $T_{1, i}$ and $T_{2, i}$ we get the following conditions:

$$
\begin{aligned}
& H_{. i}=M_{1}\left(U_{1} H_{-1, i}\right) \oplus R_{1, i}, \quad H_{i .}=M_{2}\left(U_{2} H_{i,-1}\right) \oplus R_{2, i}, \\
& H_{i}^{+}=M_{1}^{+}\left(U_{1} H_{-1, i}\right) \oplus R_{1, i}, \quad H_{i,}^{+}=M_{2}^{+}\left(U_{2} H_{i,-1}\right) \oplus R_{2, i}, \\
& H_{a}^{+}=H_{0, i} \oplus M_{1}^{+}\left(H_{1, i}\right), \quad H_{i .}^{+}=H_{i, 0} \oplus M_{2}^{+}\left(H_{i, 1}\right), \\
& P_{1, i} M_{1}^{+}\left(H_{1, i}\right) \subset M_{1}^{+}\left(U_{1} H_{-1, i}\right), \quad P_{2, i} M_{2}^{+}\left(H_{i, 1}\right) \subset M_{2}^{+}\left(U_{2} H_{i,-1}\right),
\end{aligned}
$$

Now we need some definitions. Let $L_{--}, L_{-+}, L_{+-}$and $L_{++}$be defined as follows: $\dot{L}_{--}=U_{1} U_{2} H_{-1,-1}, \quad L_{-+}=U_{1} H_{-1,1}, \quad L_{+-}$ $=U_{2} H_{1,-1}, L_{++}=H_{1,1}$ and let $\Phi_{1-}$ be a unitary map of $M_{1}\left(L_{-+}\right)$onto

$$
L_{1}^{2}\left(L_{-+}\right)=\left\{f: \Gamma \dot{\rightarrow} L_{-+} \mid \int_{\Gamma}\left\|f\left(z_{1}\right)\right\|^{2} d m\left(z_{1}\right)<\infty\right\}
$$

such that $\Phi_{1}-\left(, \sum_{n=-}^{\infty} U_{1}^{n} x_{n}\right)=\sum_{n=-x}^{\infty} z_{1}^{n} x_{n}$ where $x_{n} \in L_{-+}, \Phi_{2-}$ be a unitary map of $M_{2}\left(L_{+-}\right)$onto $L_{2}^{2}\left(L_{+-}\right)$such that $\Phi_{2-}\left(\sum_{n=-x}^{x} U_{2}^{n} x_{n}\right)=\sum_{n=-x}^{x} z_{2}^{n} x_{n}$ where $x_{n} \in L_{+-}, \Phi_{i+}$ be a unitary map of $M_{i}\left(L_{++}\right)$onto $L_{i}^{2}\left(L_{++}\right)$such that $\Phi_{i+}\left(\sum_{n=-\infty}^{\infty} U_{i}^{n} x_{n}\right)=\sum_{n=-\infty}^{\infty} z_{i}^{n} x_{n}$ where $x_{n} \in L_{++}(i=1,2)$.

Since $P_{1,1}$ commutes with $U_{1}$ and $P_{2,1}$ commutes with $U_{2}$, then by applying (2.7) and Lemma V.3.1 of [1] to $U_{1 \mid M_{1}\left(L_{-}+\right)}, U_{1 \mid M_{1}\left(L_{+}\right)}$, $P_{1,1 \mid M_{1}\left(L_{++}\right)}$and $U_{2 \mid M_{2}\left(L_{+-}\right)}, U_{2 \mid M_{2}\left(L_{+}\right)}, P_{2,1 \mid M_{2}\left(L_{+}\right)}$respectively we conclude that there are bounded analytic functions $\left\{Q_{1}^{+}\left(z_{1}\right), L_{++}, L_{-+}\right\}$and $\left\{Q_{2}^{+}\left(z_{2}\right), L_{++}, L_{+-}\right\}$such that

$$
\begin{aligned}
& \Phi_{i-} P_{i, 1} x=Q_{i}^{+} \Phi_{i+} x \text { for every } x \in M_{i}\left(L_{++}\right) \text {where for every } \\
& f \in L_{i}^{2}\left(L_{++}\right),\left(Q_{i}^{+} f\right)\left(z_{i}\right)=Q_{i}^{+}\left(z_{i}\right) f\left(z_{i}\right)(i=1,2) .
\end{aligned}
$$

Define now $\tilde{T}_{1}=P_{H_{0} .} U_{1 \mid H_{0}}, T_{2}=P_{H_{.0}} U_{2 \mid H_{0}}, P_{1}=\bigoplus_{i=-\infty}^{\infty} P_{1, i}$ and $P_{2}$ $=\bigoplus_{i=-\infty}^{\infty} P_{2, i}$. Then we can prove the following: 
LeMma 3. Suppose that $T_{1}$ and $T_{2}$ are doubly commuting contractions on $H$. Let $U_{1}, U_{2}$ be a unitary dilation of $T_{1}, T_{2}$ as in Theorem 0 . Then the following conditions are fulfilled:

(3.2) $K_{1}^{+}$is invariant subspace for $U_{1}$ and reduces $U_{2}$, $K_{2}^{+}$is invariant subspace for $U_{2}$ and reduces $U_{1}$,

$$
\tilde{T}_{1}=\bigoplus_{i=-\infty}^{\infty} T_{1, i} \text { and } \tilde{T}_{2}=\bigoplus_{i=-\infty}^{\infty} T_{2, l} \text {, }
$$

(3.4) $U_{i}$ is the minimal unitary dilation of $\tilde{T}_{i}(i=1,2)$,

(3.5) $L_{T_{1}}^{+}=H_{1 .}, L_{T_{1}}=H_{-1 .,} L_{T_{2}}^{+}=H_{.1}, L_{T_{2}}=H_{.-1}$,

(3.6) $P_{1}$ is the orthogonal projection of $K$ onto $M_{1}\left(H_{-1}\right)$,

$P_{2}$ is the orthogonal projection of $K$ onto $M_{2}\left(H_{-1}\right)$.

Proof. It is a consequence of (1.1) that for $i \neq 0, H_{i, 1}$ is a wandering subspace for $U_{2}$. Combining this with (1.2) we get that $H_{i}$ is a wandering subspace for $U_{2}$. The rest of (3.1) we obtain analogically.

Since $K_{1}^{+}=\bigoplus_{j=-\infty}^{\infty} H_{j}^{+}=\bigoplus_{i=0}^{\infty} H_{i}$ and $K_{2}^{+}=\bigoplus_{i=-\infty}^{\infty} H_{i}^{+}=\bigoplus_{j=0}^{\infty} H_{. j}$, then $(3,2)$ follows immediately by (1.3) and (1.4).

Since $P_{H_{0}} y=P_{H_{0, i}} y$ for $y \in H_{*}$, and for $x \in H_{0, i}, U_{1} x \in H_{* i}$ (see (1.4)) we have $T_{1} x=P_{H_{0} .} U_{1} x=P_{H_{0, i}} U_{1} x=T_{1, i} x$ for $x \in H_{0, i}$ which proves (3.3).

Since minimal unitary dilation of the orthogonal sum of contractions is the orthogonal sum of their minimal unitary dilations (see [1]) then (2.1) and (3.3) imply. (3.4).

It is easy to see that (3.5) is a consequence of (2.1) (2.3) and (3.3).

To prove (3.6) we have to show that $x \in M_{1}\left(H_{-1}\right.$ ) if and only if $P_{1} x$ $=x$. Let $x \in M_{1}\left(H_{-1 .}\right)$. This implies that $x=\sum_{n=-\infty}^{\infty} U_{1}^{n} x_{n}$ where $x_{n} \in H_{-1 .}$. It follows that $x_{n}=\sum_{m=-x}^{\infty} x_{n, m}$ where $x_{n, m} \in H_{-1, m}$ and consequently $x$ $=\sum_{n, m=-x}^{x} U_{1}^{n} x_{n, m}$. $\quad$ Let $\quad y_{m}=\sum_{n=-\infty}^{x} U_{1}^{n} x_{n, m} . \quad$ Since $\quad x_{n, m} \in H_{-1, m}$, $y_{m} \in M_{1}\left(H_{-1, m}\right) \subset H_{a m}$. Now $P_{1} y_{m}=P_{1 \mid H_{a m}} y_{m}=P_{1, m} y_{m}=y_{m}$, by definition of $P_{1, m}$. Thus $P_{1} x=P_{1}\left(\sum_{m=-x}^{x} y_{m}\right)=\sum_{m=-\infty}^{\infty} P_{1} y_{m}=\sum_{m=-x}^{\infty} y_{m}=x$. Suppose now that $P_{1} x=x$. Every $x \in K$ is of the form $x=\sum_{m=-\infty}^{\infty} y_{m}$ where $y_{m} \in H_{-m}$. Our assumption implies that $p_{1, m} y_{m}=y_{m}$. It follows, by definition of $P_{1, m}$ 
that $y_{m} \in M_{1}\left(H_{-1, m}\right)$, hence $y_{m}=\sum_{n=-x}^{\infty} U_{1}^{n} x_{n, m}$ where $x_{n, m} \in H_{-1, m}$. Consequently, $\quad x=\sum_{n, m=-\gamma}^{\infty} U_{1}^{n} x_{n, m}$. Since for every $n$ the sum $\sum_{m=-\infty}^{\infty} x_{n, m} \in H_{-1 .}$, we get that $x \in M_{1}\left(H_{-1 .}\right)$ and the proof of Lemma 3 is complete.

Now, applying Theorems I.1.4 and I.2.1 of [1] by Lemma 3 to the operators $\tilde{T}_{1}$ and $\widetilde{T}_{2}$ we get the following conditions

$$
\begin{array}{lc}
\text { (3.7) } K=M_{1}\left(U_{1} H_{-1}\right) \oplus R_{1}, \quad K=M_{2}\left(U_{2} H_{.-1}\right) \oplus R_{2}, \\
\text { (3.8) } K_{1}^{+}=M_{1}^{+}\left(U_{1} H_{-1}\right) \oplus R_{1}, \quad K_{2}^{+}=M_{2}^{+}\left(U_{2} H_{.-1}\right) \oplus R_{2}, \\
\text { (3.9) } K_{1}^{+}=H_{0 .} \oplus M_{1}^{+}\left(H_{1}\right), \quad K_{2}^{+}=H_{.0} \oplus M_{2}^{+}\left(H_{.1}\right), \\
\text { (3.10) } P_{1} M_{1}^{+}\left(H_{1}\right) \subset M_{1}^{+}\left(U_{1} H_{-1 .}\right), \quad P_{2} M_{2}^{+}\left(H_{.1}\right) \subset M_{2}^{+}\left(U_{2} H_{.-1}\right), \\
\text { (3.11) if } T_{1} \text { is completely nonunitary then } K=M_{1}\left(U_{1} H_{-1 .}\right) \vee M_{1}\left(H_{1 .}\right), \\
\text { if } T_{2} \text { is completely nonunitary then } K=M_{2}\left(U_{2} H_{.-1}\right) \vee M_{2}\left(H_{.1}\right), \\
\text { (3.12) if } T_{1} \text { is completely nonunitary then } R_{1}=\frac{\left(I-P_{1}\right) M_{1}\left(H_{1}\right)}{\left(I-P_{2}\right) M_{2}\left(H_{.1}\right) .}
\end{array}
$$

Now we define the following operators $\tilde{T}_{1,0}=U_{2} T_{1,-1} U_{2 \mid U_{2} H_{0,-1}}^{-1}$ and $T_{2,0}=U_{1} T_{2,-1} U_{1 \mid U_{1} H_{-1,0}}^{-1}$. We prove the lemma:

LEMMA 4. Suppose that $T_{1}$ and $T_{2}$ are doubly commuting contractions on $H$. Let $U_{1}, U_{2}$ be a unitary dilation of $T_{1}, T_{2}$ as in Theorem 0 . Then with notations as above the following conditions hold:

(4.3) $L_{--}, L_{+-}$and $L_{-}, L_{-}$are wandering subspaces for $U_{1}$ and $U_{2}$ respectively,

(4.4) $P_{1 \mid U_{1} H_{--1}}$ is the orthogonal projection of $U_{2} H_{.-1}$ onto $M_{1}\left(L_{--}\right)$,

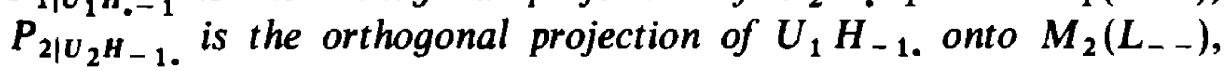

(4.5) if $T_{1}$ is completely nonunitary then $\tilde{T}_{i, 0}$ is completely nonunitary $(i=1,2)$,

$$
\begin{array}{ll}
L_{T_{1,0}}^{+}=L_{+-}, & L_{T_{1,0}}^{+}=U_{2} H_{-1,-1}, \\
L_{T_{2,0}}^{+}=L_{-+}, & L_{\tau_{2,0}}=U_{1} H_{-1,-1} .
\end{array}
$$

Proof. Since $H_{-1}$ reduces $U_{1}$ and $P_{1}$ (see (1.4) and definition of $\left.P_{1}\right)$ we have $U_{1} U_{2} H_{.-1}=U_{2} U_{1} H_{.-1}=U_{2} H_{.-1}$ and $P_{1} U_{2} H_{.-1}$ 
$=U_{2} P_{1} H_{.-1} \subset U_{2} H_{.-1}$. The last equality is true because $P_{1}$ is the orthogonal projection onto $M_{1}\left(H_{-1}\right)$ and $M_{1}\left(H_{-1}\right)$ reduces $U_{2}$. Since $U_{2}$ is unitary and $P_{1}$ is selfadjoint, the proof of (4.1) is complete.

Equality $P_{1} U_{2} H_{0-1}=U_{2} P_{1} H_{.-1}$ gives $P_{1} U_{2} H_{0-1}=U_{2} M_{1}\left(H_{-1,-1}\right)$ $=M_{1}\left(U_{2} H_{-1,-1}\right)$. This implies (4.4).

Let $P_{H_{0,-1}}$ be the projection of $H_{-, 1}$ onto $U_{1} H_{0,-1}$ and let $P_{v_{2} H_{0,-1}}$ be the projection of $U_{2} H_{--1}$ onto $U_{2} H_{0,-1}$. It is easy to see that for $x \in$ $U_{2} H_{0,-1}$ we have $P_{U_{2} H_{0,-1}} x=U_{2} P_{H_{0,-1}} U_{2}^{-1} x$. Suppose now that $x \in$ $U_{2} H_{0,-1}$. Then $x=U_{2} y$ where $y \in H_{0,-1}$ and for every $n \geqslant 0 U_{1}^{n} x \in U_{2} H_{-1}$ (see (1.4)). Now, by (2.1) we get $T_{1,0}^{n} x=U_{2} T_{1,-1}^{n} U_{2}^{-1} x=U_{2} T_{1,-1}^{n} y=$ $U_{2} P_{H_{0,-1}} U_{1}^{n} y=U_{2} P_{H_{0,-1}} U_{1}^{n} U_{2}^{-1} x=U_{2} P_{H_{0,-1}} U_{2}^{-1} U_{1}^{n} x=P_{U_{2} H_{0,-1}} U_{1}^{n} x$ which proves that $U_{1 \mid U_{2} H_{0,-1}}$ is a dilation of $\tilde{T}_{1,0}$. Since $\bigvee_{n=-\infty}^{\infty} U_{1}^{n} U_{2} H_{0,-1}$ $=U_{2}\left(\bigvee_{n=-\infty}^{\infty} U_{1}^{n} H_{0,-1}\right)=U_{2} H_{.-1}$ (by (2.1)), (4.2) is proved.

As an immediate consequence of (1.1) we get (4.3).

If $T_{i}$ is completely nonunitary then by (2.2) $T_{i,-1}$ is completely nonunitary. Since $\tilde{T}_{i, 0}$ is unitarily equivalent to $T_{i,-1}$ we get (4.5).

It is easy to see that $L_{T_{1,0}}^{+}=U_{2} L_{T_{1,-1}}^{+}$. Then by (2.3) we have $L_{T_{1,0}}^{+}$ $=L_{+-}$. The rest of (4.6) we prove analogically. This completes the proof of Lemma 4.

Now applying Theorems I.1.4 and I.2.1 of [1] to the operators $\tilde{T}_{1,0}$ and $\tilde{T}_{2,0}$, we get by Lemma 4 the following conditions:

$$
\begin{aligned}
U_{2} H_{\cdot-1}= & M_{1}\left(L_{--}\right) \oplus \tilde{R}_{1}, \quad U_{1} H_{-1 .}=M_{2}\left(L_{--}\right) \oplus \tilde{R}_{2}, \\
U_{2} H_{--1}^{+}= & M_{1}^{+}\left(L_{--}\right) \oplus \tilde{R}_{1}, \quad U_{1} H_{-1 .}^{+}=M_{2}^{+}\left(L_{--}\right) \oplus \tilde{R}_{2}, \\
& U_{2} H_{0^{+}-1}^{+}=U_{2} H_{0,-1} \oplus M_{1}^{+}\left(L_{+-}\right), \\
& U_{1} H_{-1 .}^{+}=U_{1} H_{-1,0} \oplus M_{2}^{+}\left(L_{-+}\right), \\
& P_{1 \mid H_{2} H_{\bullet-1}} M_{1}^{+}\left(L_{+-}\right) \subset M_{1}^{+}\left(L_{--}\right), \\
& P_{2 \mid U_{1} H_{-1 .}} M_{2}^{+}\left(L_{-+}\right) \oplus M_{2}^{+}\left(L_{--}\right),
\end{aligned}
$$

$$
\begin{aligned}
& \text { if } T_{1} \text { is completely nonunitary then } U_{2} H_{0-1} \\
& =M_{1}\left(L_{--}\right) \vee M_{1}\left(L_{+-}\right) \text {, } \\
& \text { if } T_{2} \text { is completely nonunitary then } U_{1} H_{-1} \text {. } \\
& =M_{2}\left(L_{-}\right) \vee M_{2}\left(L_{-}+\right) \text {, }
\end{aligned}
$$

(4.12) if $T_{1}$ is completely nonunitary then $\tilde{R}_{1}=\overline{\left(I-P_{1}\right) M_{1}\left(L_{+-}\right)}$,

if $T_{2}$ is completely nonunitary then $\tilde{R}_{2}=\overline{\left(I-P_{2}\right) M_{2}\left(L_{-+}\right)}$. 
Therefore we obtain that there are bounded analytic functions $\left\{Q_{1}^{-}\left(z_{1}\right), L_{+-}, L_{--}\right\}$and $\left\{Q_{2}^{-}\left(z_{2}\right), L_{-+}, L_{--}\right\}$such that

(4.13) $\tilde{\Phi}_{1-} P_{1} x=Q_{1}^{-} \widetilde{\Phi}_{1+} x$ for every $x \in M_{1}\left(L_{+-}\right)$where for every $f \in L_{1}^{2}\left(L_{+-}\right)$we have $\left(Q_{1}^{-} f\right)\left(z_{1}\right)=Q_{1}^{-}\left(z_{1}\right) f\left(z_{1}\right)$,

$\Phi_{2-} P_{2} x=Q_{2}^{-} \tilde{\Phi}_{2+} x$ for every $x \in M_{2}\left(L_{-+}\right)$where for every $f \in L_{2}^{2}\left(L_{-+}\right)$we have $\left(Q_{2}^{-} f\right)\left(z_{2}\right)=Q_{2}^{-}\left(z_{2}\right) f\left(z_{2}\right)$.

$\tilde{\Phi}_{i-}$ and $\tilde{\Phi}_{i+}$ are defined as follows:

$\tilde{\Phi}_{i-}$ is a unitary map of $M_{i}\left(L_{--}\right)$onto $L_{i}^{2}\left(L_{--}\right)$such that $\tilde{\Phi}_{i-}\left(\sum_{n=-\infty}^{\infty} U_{i}^{n} x_{n}\right)=\sum_{n=-\infty}^{\infty} z_{i}^{n} x_{n}$ with $x_{n} \in L_{--}(i=1,2)$;

$\tilde{\Phi}_{1+}^{n=-\infty}$ is a unitary map of $M_{1}\left(L_{+-}\right)$onto $L_{1}^{2}\left(L_{+-}\right)$such that $\tilde{\Phi}_{1+}\left(\sum_{n=-\infty}^{\infty} U_{1}^{n} x_{n}\right)=\sum_{n=-\infty}^{\infty} z_{1}^{n} x_{n}$ with $x_{n} \in L_{+-}$;

$\Phi_{2+}$ is a unitary map of $M_{2}\left(L_{-+}\right)$onto $L_{2}^{2}\left(L_{-+}\right)$such that $\tilde{\Phi}_{2+}\left(\sum_{n=-\infty}^{\infty} U_{2}^{n} x_{n}\right)=\sum_{n=-\infty}^{\infty} z_{2}^{n} x_{n}$ with $x_{n} \in L_{-+}$.

Our next result is the following:

Lemma 5. Suppose that $T_{1}$ and $T_{2}$ are doubly commuting contractions on $H$. Let $U_{1}, U_{2}$ be unitary dilations of $T_{1}, T_{2}$ as in Theorem 0 . Then the following conditions hold true:

(5.1) if $L$ is a wandering subspace for the pair $U_{1}, U_{2}$ then $M_{1}(L)$ and $M_{2}(L)$ are wandering subspaces for $U_{2}$ and $U_{1}$ respectively and $M(L)$ $=M_{1} M_{2}(L)=M_{2} M_{1}(L)$,

(5.2) if $T_{1}$ and $T_{2}$ are completely nonunitary then

$$
K=M\left(H_{-1,-1}\right) \vee M\left(H_{-1,1}\right) \vee M\left(H_{1,-1}\right) \vee M\left(H_{1,1}\right),
$$

(5.3) $P_{1}$ commutes with $U_{2}$ and $P_{1} ; P_{2}$ commutes with $U_{1}$,

$$
\begin{gathered}
\left(I-P_{1}\right) K_{2}^{+} \subset K^{+}, \quad\left(I-P_{2}\right) K_{1}^{+} \subset K^{+}, \\
\left(I-P_{1}\right) M_{1}\left(H_{1}^{+}\right) \subset K^{+}, \quad\left(I-P_{2}\right) M_{2}\left(H_{.1}^{+}\right) \subset K^{+}, \\
\left(I-P_{1}\right)\left(I-P_{2}\right) K \subset K^{+}, \\
P_{1} P_{2} K=M\left(L_{--}\right), \\
P_{1}\left(I-P_{2}\right) K=\overline{\left(I-P_{2}\right) M_{1} M_{2}\left(L_{-+}\right)}, \\
P_{2}\left(I-P_{1}\right) K=\overline{\left(I-P_{1}\right) M_{2} M_{1}\left(L_{+-}\right)} \\
\left(I-P_{1}\right)\left(I-P_{2}\right) K=\overline{\left(I-P_{1}\right)\left(I-P_{2}\right) M\left(L_{++}\right)},
\end{gathered}
$$




$$
\begin{aligned}
& K=M\left(L_{--}\right) \oplus \overline{\left(I-P_{1}\right) M_{1} M_{2}\left(L_{-+}\right)} \oplus \overline{\left(I-P_{2}\right) M_{1} M_{2}\left(L_{+-}\right)} \oplus \overline{\left(I-P_{1}\right)\left(I-P_{2}\right) M\left(L_{++}\right)}, \\
& P_{i} K^{+} \subset K^{+}, \quad\left(I-P_{i}\right) K^{+} \subset K^{+} \quad(i=1,2), \\
& K^{+}=M^{+}\left(L_{--}\right) \oplus \overline{\left(I-P_{1}\right) M_{1} M_{2}^{+}\left(L_{-+}\right)} \oplus \\
& \oplus \overline{\left(I-P_{2}\right) M_{1}^{+} M_{2}\left(L_{+-}\right)} \oplus \overline{\left(I-P_{1}\right)\left(I-P_{2}\right) M\left(L_{++}\right)}, \\
& K^{+} \ominus H=M^{+}\left(L_{-+}\right) \vee M^{+}\left(L_{+-}\right) \vee \overline{\left(I-P_{1}\right) M_{1} M_{2}^{+}\left(L_{++}\right)} \vee \\
& \vee \overline{\left(I-P_{2}\right) M_{1}^{+} M_{2}\left(L_{++}\right)} .
\end{aligned}
$$

Proof. It is easy to see that to prove (5.1) it is sufficient to show that $M_{1}(L)$ is wandering for $U_{2}$. Let $n \neq 0$ and $x, y \in M_{1}(L)$. The vectors $x$ and $y$ have the form $x=\sum_{m=-\infty}^{\infty} U_{1}^{m} x_{m}, y=\sum_{k=-\infty}^{\infty} U_{1}^{k} y_{k}$ where $x_{m}, y_{k} \in L$. It follows that $\left(U_{2}^{n} x, y\right)=\sum_{k, m=-\infty}^{\infty}\left(U_{2}^{n} U_{1}^{m} x_{m}, U_{1}^{k} y_{k}\right)=\sum_{k, m=-\infty}^{\infty}\left(U_{2}^{n} U_{1}^{m-k} x_{m}, y_{k}\right)$. Evidently for every $m, k,|n|+|m-k|>0$. Since $L$ is a wandering subspace for the pair $U_{1}, U_{2}$, we have $\left(U_{2}^{n} U_{2}^{m-k} x_{m}, y_{k}\right)=0$ and consequently $\left(U_{2}^{n} x, y\right)=0$ which completes the proof of $(5.1)$.

If $T_{1}$ is completely nonunitary then by (2.8) we have $H_{.1}$ $=M_{1}\left(H_{-1,1}\right) \vee M_{1}\left(H_{1,1}\right)$ and $H_{.-1}=M_{1}\left(H_{-1,-1}\right) \vee M_{1}\left(H_{1,-1}\right)$. Then, if, additionally, $T_{2}$ is completely nonunitary, by (3.11) we have

$$
K=M_{2}\left(M_{1}\left(H_{-1,-1}\right) \vee M_{1}\left(H_{1,-1}\right)\right) \vee M_{2}\left(M_{1}\left(H_{-1,1}\right) \vee M_{1}\left(H_{1,1}\right)\right) .
$$

Now by (5.1) we get (5.2).

Since $H_{-1}$, reduces $U_{2}$ (see (1.4)) and $U_{2}$ doubly commutes with $U_{1}$, we see that $M_{1}\left(H_{-1}\right.$. $)$ reduces $U_{2}$. Thus, by (3.6) we may conclude that $P_{1}$ commutes with $U_{2}$. Analogically we prove that $P_{2}$ commutes with $U_{1}$. Now by definition of $P_{1}$ we have that $H_{-1}$, reduces $P_{1}$. Since $U_{2}$ commutes with $P_{1}$, we have that $M_{2}\left(H_{0-1}\right)$ reduces $P_{1}$. Hence (3.6) gives us that $P_{1}$ and $P_{2}$ commute which completes the proof of (5.3). have

Since $\left(I-P_{1}\right)=\bigoplus_{i=-\infty}^{\infty}\left(I-P_{1, i}\right)$ and $K_{2}^{+}=\bigoplus_{i=0}^{\infty} H_{s i}$, by (2.4) and (2.5) we

$$
\left(I-P_{1}\right) K_{2}^{+}=\bigoplus_{i=0}^{\infty}\left(I-P_{1, i}\right) H_{d}=\bigoplus_{i=0}^{\infty} R_{1, i} \subset \bigoplus_{i=0}^{\infty} H_{. i}^{+}=K^{+}
$$

which proves (5.3). Since $H_{1}^{+} \subset K^{+} \subset K_{2}^{+}$and $K_{2}^{+}$reduces $U_{1}, M_{1}\left(H_{1}^{+}\right) \subset K_{2}^{+}$. Now (5.4) implies (5.5).

It is a consequence of (5.4) that to prove (5.6) it is sufficient to show that $\left(I-P_{2}\right) K \subset K_{2}^{+}$. But this follows from (3.6), (3.7), and (3.8).

By definition of $P_{2}$ we. have that $P_{2} H_{-1 .}=P_{2,-1} H_{-1 .}=M_{2}\left(H_{-1,-1}\right)$. 
It follows, by the commutativity of $P_{2}$ and $U_{1}$ that $P_{2} M_{1}\left(H_{-1}\right)$ $=M_{1}\left(P_{2} H_{-1}\right)=M_{1} M_{2}\left(H_{-1,-1}\right)=M\left(H_{-1,-1}\right)=M\left(L_{--}\right)$. Since $P_{2} K$ $=M_{1}\left(H_{-1}\right)$, see (3.6), we have $P_{1} P_{2} K=P_{2} P_{1} K=P_{2} M_{1}\left(H_{-1}\right)=M\left(L_{--}\right)$ which proves (5.7).

By (3.6) we have $P_{1} K=M_{1}\left(H_{-1}\right)$. Thus

$$
P_{1}\left(I-P_{2}\right) K=\left(I-P_{2}\right) P_{1} K=M_{1}\left(\left(I-P_{2}\right) H_{-1 .}\right) \text {. }
$$

If $T_{2}$ is completely nonunitary, then by (2.9) we have

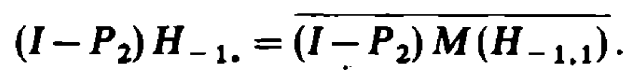

Consequently

$$
P_{1}\left(I-P_{2}\right) K=\overline{\left(I-P_{2}\right) M_{1} M_{2}\left(H_{-1,1}\right)}=\overline{\left(I-P_{2}\right) M_{1} M_{2}\left(L_{-+}\right)}
$$

which ends the proof of (5.8). If $T_{2}$ is completely nonunitary, by (3.12) we have

$$
\left(I-P_{1}\right)\left(I-P_{2}\right) K=\overline{\left(I-P_{1}\right)\left(I-P_{2}\right) M_{2}\left(H_{.1}\right)} .
$$

If additionally $T_{1}$ is completely nonunitary then by (2.9),

$$
\left(I-P_{1}\right) H_{.1}=\overline{\left(I-P_{1}\right) M_{1}\left(H_{1,1}\right)} \text {. }
$$

Consequently

$$
\left(I-P_{1}\right)\left(I-P_{2}\right) K=\overline{\left(I-P_{1}\right)\left(I-P_{2}\right) M\left(L_{++}\right)}
$$

which proves (5.9).

Now, by (5.7), (5.8), (5.9), the evident equality

$$
K=P_{1} P_{2} K \oplus P_{1}\left(I-P_{2}\right) K \oplus P_{2}\left(I-P_{1}\right) K \oplus\left(I-P_{1}\right)\left(I-P_{2}\right) K
$$

implies (5.10).

Since $K^{+} \subset K_{2}^{+}$, by (5.4) we have $\left(I-P_{1}\right) K^{+} \subset K^{+}$. To prove inclusion $P_{1} K^{+} \subset K^{+}=\bigoplus_{i=0}^{\infty} H_{d}^{+}$it is sufficient to show that $P_{1} H_{d}^{+} \subset K^{+}$for every $i \geqslant 0$. But $H_{j}^{+}=M_{1}^{+}\left(U_{1} H_{-1, i}\right) \oplus\left(I-P_{1, i}\right) H_{.1}$ (see (2.5) and (2.4)). Hence $P_{1} H_{o i}^{+}=M_{1}^{+}\left(U_{1} H_{-1, i}\right) \subset H_{i}^{+} \subset K^{+}$. So we have proved (5.11). Since $H_{d i}^{+}$is invariant for $U_{1}$ and $H_{i .}^{+}$is invariant for $U_{2}$ (see (1.3)), $K^{+}=\bigoplus_{i=0}^{a} H_{d}^{+}$ $=\bigoplus_{i=0}^{\chi} H_{i^{+}}^{+}$is invariant for $U_{1}$ and $U_{2}$. By $(0.2)$ we have that $L_{--}$ $=U_{1} U_{2} H_{-1,-1} \subset K^{+}$. Consequently for every $n, m \geqslant 0, U_{1}^{n} U_{2}^{m} L_{--} \subset K^{+}$ which proves that $M^{+}\left(L_{--}\right) \subset K^{+}$. Since $M_{1} M_{2}^{+}\left(L_{+-}\right)=M_{2}^{+} M_{1}\left(L_{+-}\right)$ $\subset M_{2}^{+}\left(H_{.-1}\right) \subset K_{2}^{+}$(see (3.8)), by (5.4) we get that $\left(I-P_{1}\right) M_{1} M_{2}^{+}\left(L_{+-}\right)$ $\subset K^{+}$. Analogically we prove that $\left(I-P_{2}\right) M_{1}^{+} M_{2}\left(L_{-+}\right) \subset K^{+}$. Finally by $(5.6)$ 
we get that

$$
\begin{aligned}
M^{+}\left(L_{--}\right) \vee\left(I-P_{1}\right) M_{1} M_{2}^{+}\left(L_{+-}\right) \vee & \left(I-P_{2}\right) M_{1}^{+} M_{2}\left(L_{-+}\right) \vee \\
& \vee\left(I-P_{1}\right)\left(I-P_{2}\right) M\left(L_{++}\right) \subset \dot{K}^{+} .
\end{aligned}
$$

Suppose now that $x \in K^{+}$. Then $x=x_{1}+x_{2}+x_{3}+x_{4}$ where $x_{1}=P_{1} P_{2} x$, $x_{2}=P_{1}\left(I-P_{2}\right) x, x_{3}=P_{2}\left(I-P_{1}\right) x$ and $x_{4}=\left(I-P_{1}\right)\left(I-P_{2}\right) x$. It follows, by (5.11), that $x_{i} \in K^{+}(i=1, \ldots, 4)$. This implies that $x_{1} \perp U_{1}^{n} U_{2}^{m} L_{-}$if $n<0$ or $m<0$. But $x_{1}=P_{1} P_{2} x \in P_{1} P_{2} K=M\left(L_{--}\right)$(see (5.7)). Consequently $x_{1} \in M^{+}\left(L_{--}\right)$. Since $x_{2} \in K^{+},\left(I-P_{2}\right) x_{2}=x_{2} \in U_{1}^{n} U_{2}^{m} L_{+}+$if $n<0$. It follows that $x_{2} \perp\left(I-P_{2}\right) U_{1}^{n} U_{2}^{m} L_{++}$if $m<0$. But $x_{2}=P_{1}\left(I-P_{2}\right) x \in P_{1}\left(I-P_{2}\right)$, $K=\left(I-P_{2}\right) M\left(L_{++}\right)$. Hence $x_{2} \in\left(I-P_{2}\right) M_{1} M_{2}^{+}\left(L_{++}\right)$. Analogically we prove that $x_{3} \in\left(I-P_{1}\right) M_{2}^{+} M_{1}\left(L_{+-}\right)$. Finally

$$
x_{4}=\left(I-P_{1}\right)\left(I-P_{2}\right) x \in\left(I-P_{1}\right)\left(I-P_{2}\right) K=\left(I-P_{1}\right)\left(I-P_{2}\right) M\left(L_{++}\right) .
$$

We have proved that

$$
\begin{aligned}
K^{+}=M^{+}\left(L_{--}\right) \vee\left(I-P_{1}\right) M_{1} M_{2}^{+}\left(L_{+-}\right) \vee & \left(I-P_{2}\right) M_{1}^{+} M_{2}\left(L_{-+}\right) \vee \\
& \vee\left(I-P_{1}\right)\left(I-P_{2}\right) M\left(L_{++}\right) .
\end{aligned}
$$

Now, (5.12) is an easy consequence of (5.10).

Since $M_{1} M_{2}^{+}\left(L_{++}\right)=M_{2}^{+} M_{1}\left(L_{++}\right) \subset M_{2}^{+}\left(H_{.1}\right)=\bigoplus H_{a i} \subset K_{2}^{+}$, by (5.4) we get that $\left(I-P_{1}\right) M_{1} M_{2}^{+}\left(L_{++}\right) \subset K^{+}$. Moreover, $\left(I-P_{1}\right) M_{1} M_{2}^{+}\left(L_{++}\right)$ $\subset\left(I-P_{1}\right) \oplus H_{\alpha i} \subset \oplus H_{\alpha_{i}}$. If follows that $H \perp\left(I-P_{1}\right) M_{1} M_{2}^{+}\left(L_{++}\right)$. Analogically we prove that $\left(I-P_{2}\right) M_{1}^{+} M_{2}\left(L_{++}\right) \subset K^{+}$and $H \perp(I-$ $\left.-P_{2}\right) M_{1}^{+} M_{2}\left(L_{++}\right)$. By $(0.2)$ we have that $L_{-+} \subset K^{+}$and $L_{+-} \subset K^{+}$. But $K^{+}$ is invariant for $U_{1}$ and $U_{2}$, hence $M^{+}\left(L_{-+}\right) \subset K^{+}$and $M^{+}\left(L_{+-}\right) \subset K^{+}$. Since $M^{+}\left(L_{-}+\right)=M_{2}^{+} M_{1}^{+}\left(L_{-+}\right) \subset M_{2}^{+}\left(H_{.1}\right)=\bigoplus H_{\alpha_{j}}$ and $M^{+}\left(L_{+--}\right) \subset M_{1}^{+}\left(H_{1 .}\right)$ $=\bigoplus H_{i}$, we have that $H \perp M^{+}\left(L_{-+}\right)$and $H \perp M^{+}\left(L_{+-}\right)$. We have proved that

$$
\begin{aligned}
& M^{+}\left(L_{-+}\right) \vee M^{+}\left(L_{+-}\right) \vee\left(I-P_{1}\right) M_{1} M_{2}^{+}\left(L_{++}\right) \vee \\
& \vee\left(I-P_{2}\right) M_{1}^{+} M_{2}\left(L_{++}\right) \subset K^{+} \ominus H .
\end{aligned}
$$

To finish the proof, it is sufficient to show that for every $i>1, H_{\alpha}^{+}$and $H_{i \text {. }}^{+}$ are included in

$$
M^{+}\left(L_{+-}\right) \vee M^{+}\left(L_{-+}\right) \vee\left(I-P_{1}\right) M_{1} M_{2}^{+}\left(L_{+} L_{+}\right) \vee\left(I-P_{2}\right) M_{1}^{+} M_{2}\left(L_{++}\right) .
$$

Since for $i>1, H_{-i}^{+}=U_{2}^{i-1} H_{.1}^{+}$and the right-hand of inclusion which we have to prove is invariant for $U_{2}$, it is sufficient to show that

$$
\begin{aligned}
H_{.1}^{+} \subset M^{+}\left(L_{-+}\right) \vee M^{+}\left(L_{+-}\right) \vee\left(I-P_{1}\right) M_{1} M_{2}^{+}\left(L_{++}\right) \vee & \\
& \vee\left(I-P_{2}\right) M_{1}^{+} M_{2}\left(L_{++}\right) .
\end{aligned}
$$


Since $T_{1}$ is completely nonunitary, by (2.5) and (2.9) we have

$$
\begin{aligned}
H_{.1}^{+}=M_{1}^{+}\left(L_{-+}\right) \oplus\left(I-P_{1}\right) M_{1}\left(L_{++}\right) & \\
\subset M_{2}^{+}\left(M_{1}^{+}\left(L_{-+}\right)\right) & \vee M_{2}^{+}\left(I-P_{1}\right) M_{1}\left(L_{++}\right) \\
& =M^{+}\left(L_{-+}\right) \vee\left(I-P_{1}\right) M_{1} M_{2}^{+}\left(L_{++}\right) .
\end{aligned}
$$

Analogically we prove that $H_{i_{0}}^{+} \subset M^{+}\left(L_{+-}\right) \vee\left(I-P_{2}\right) M_{1}^{+} M_{2}\left(L_{++}\right)$which finishes the proof of Lemma 5.

Now we need the following definitions: Let $L$ be a wandering subspace for the pair $U_{1}, U_{2}$. Then: $\Phi_{i, L}$ denotes the unitary map of $M(L)$ onto $\bigoplus M_{i}(L)$ (the orthogonal sum of countably many copies of $M_{i}(L)$ ) such that $\Phi_{i, L}\left(\sum_{n=-\infty}^{\infty} U_{j}^{n} x_{n}\right)=\left\{x_{n}\right\}$, where $x_{n} \in M_{i}(L) \quad(i=1,2) \quad(j \in\{1,2\} \backslash\{i\}), \tilde{\Phi}_{i, L}$ denotes the unitary map of $\oplus M_{i}(L)$ onto $L^{2}(L)(i=1,2)$ such that $\Phi_{1, L}\left(\left\{\sum_{n=-\infty}^{\infty} U_{1}^{n} x_{n, m}\right\}\right)=\sum_{n, m=-\infty}^{\infty} z_{1}^{n} z_{2}^{m} x_{n, m} \quad$ and $\cdot \Phi_{2, L}\left(\left\{\sum_{m=-\infty}^{\infty} U_{2}^{m} x_{n m}\right\}\right)$ $=\sum_{n, m=-\infty}^{\infty} z_{1}^{n} z_{2}^{m} x_{n, m} \quad$ where $\quad x_{n, m} L, \quad L^{2}(L)=\left\{f: \quad \Gamma^{2} \rightarrow L \quad\right.$ such that $\left.\int_{\Gamma^{2}}\left\|f\left(z_{1}, z_{2}\right)\right\|^{2} d m\left(z_{1}, z_{2}\right)<\infty\right\} . \Phi_{a b}$ denotes the unitary map of $M\left(L_{a b}\right)$ onto $L^{2}\left(L_{a b}\right)$ such that $\Phi_{a b}\left(\sum_{n, m=-\infty}^{\infty} U_{1}^{n} U_{2}^{m} x_{n, m}\right)=\sum_{n, m=-\infty}^{\infty} z_{1}^{n} z_{2}^{m} x_{n, m}$ where $x_{n m} \in L_{a b}$ $(a, b=+,-)$.

If $\Phi$ is a unitary map $L$ onto $L$, then $\oplus \Phi$ denotes the unitary map of $\oplus L$ onto $\oplus L^{\prime}$ such that $(\oplus \Phi)\left(\left\{x_{n}\right\}\right)=\left\{\Phi x_{n}\right\}$ where $x_{n} \in L$. With these definitions the following lemma is not difficult to prove:

LEMMA 6. With the notations as above the following equalities hold true:

$$
\begin{aligned}
& \Phi_{--}=\tilde{\Phi}_{2, L_{-}-} \circ \bigoplus \Phi_{2_{-}} \circ \Phi_{2, L_{-}-}=\tilde{\Phi}_{2, L_{-}} \circ \bigoplus \Phi_{1-} \circ \Phi_{1, L_{-}-} \text {, } \\
& \tilde{\Phi}_{-+}=\tilde{\Phi}_{2, L_{-}+} \circ \bigoplus \Phi_{2+} \circ \Phi_{2, L_{-}+}=\tilde{\Phi}_{1, L_{-}+} \circ \oplus \Phi_{1-} \circ \Phi_{1, L_{-}+} \text {, } \\
& \Phi_{+-}=\tilde{\Phi}_{2, L_{+}-} \circ \oplus \Phi_{2-} \circ \Phi_{2, L_{+}-}=\tilde{\Phi}_{1, L_{+}-} \circ \oplus \Phi_{1+} \circ \Phi_{1, L_{+-}} \text {, } \\
& \Phi_{++}=\tilde{\Phi}_{2, L_{+}+} \circ \oplus \Phi_{2+} \circ \Phi_{2, L_{+}+}=\tilde{\Phi}_{1, L_{+}+} \circ \oplus \Phi_{1+} \circ \Phi_{1, L_{+}+} .
\end{aligned}
$$

Suppose now that $T_{1}$ and $T_{2}$ are completely nonunitary doubly commuting contractions on $H$. Let $U_{1}, U_{2}$ be their unitary dilation as in Theorem 0. Let $x \in M\left(L_{+-}\right)$. Then $\Phi_{2, L_{-}-} P_{1} x=\left\{P_{1} x_{n}\right\}_{n=-\infty}^{\infty}$ if $x=\sum_{n=-\infty}^{\infty} U_{2}^{n} x_{n}$ where $x_{n}=\sum_{m=-\infty}^{\infty} U_{1}^{m} x_{m, n} \in M_{1}\left(L_{+-}\right) \subset U_{2} H_{-1}$. Thus, by (4.13)

$$
\begin{aligned}
\left(\bigoplus \Phi_{1-}\right)\left(\Phi_{2, L_{-}-}\left(P_{1} x\right)\right)(z) & =\left\{\left(\Phi_{1-} P_{1} x_{n m}\right)(z)\right\}_{n=-\infty}^{\infty} \\
& =\left\{Q_{1}^{-}(z)\left(\Phi_{1}+x_{n}\right)(z)\right\}_{n=-\infty}^{\infty}
\end{aligned}
$$


Consequently, by (6.1) we have

$$
\begin{aligned}
\left(\Phi_{--} P_{1} x\right)\left(z_{1}, z_{2}\right) & =\sum_{n=-\infty}^{\infty} z_{2}^{n} Q_{1}^{-}\left(z_{1}\right) \sum_{n=-\infty}^{\infty} z_{1}^{m} x_{m, n} \\
& =Q_{1}^{-}\left(z_{1}\right) \sum_{n, m=-\infty}^{\infty} z_{1}^{m} z_{2}^{n} x_{n, m}=Q_{1}^{-}\left(z_{1}\right)\left(\Phi_{+-} x\right)\left(z_{1}, z_{2}\right) .
\end{aligned}
$$

We have proved that:

(7.1) if $x \in M\left(L_{+-}\right)$then $\Phi_{--} P_{1} x=Q_{1}^{-} \Phi_{+-} x$ where for $f \in L^{2}\left(L_{+_{-}}\right)$,

$$
\left(Q_{1}^{-} f\right)\left(z_{1}, z_{2}\right)=Q_{1}^{-}\left(z_{1}\right) f\left(z_{1}, z_{2}\right) \text {. }
$$

Analogically we prove:

(7.2) if $x \in M\left(L_{-+}\right)$then $\Phi_{--} P_{2} x=Q_{2}^{-} \Phi_{-+} x$ where for $f \in L^{2}\left(L_{-+}\right)$

$$
\left(Q_{2}^{-} f\right)\left(z_{1}, z_{2}\right)=Q_{2}^{-}\left(z_{2}\right) f\left(z_{1}, z_{2}\right),
$$

(7.3) if $x \in M\left(L_{++}\right)$then $\Phi_{+-} P_{2} x=Q_{2}^{+} \Phi_{++} x$ where for $f \in L^{2}\left(L_{++}\right)$,

$$
\left(Q_{2}^{+} f\right)\left(z_{1}, z_{2}\right)=Q_{2}^{+}\left(z_{2}\right) f\left(z_{1}, z_{2}\right),
$$

(7.4) if $x \in M\left(L_{++}\right)$then $\Phi_{-+} P_{1} x=Q_{1}^{+} \Phi_{++} x$ where for $f \in L^{2}\left(L_{++}\right)$,

$$
Q_{1}^{+} f\left(z_{1}, z_{1}\right)=Q_{1}^{+}\left(z_{1}\right) f\left(z_{1}, z_{2}\right) \text {. }
$$

Now, if $x \in M\left(L_{++}\right)$then $\Phi_{--} P_{1} P_{2} x=Q_{1}^{-} \Phi_{+-} P_{2} x=Q_{1}^{-} Q_{2}^{+} \Phi_{++} x$. Suppose now, that $x \in M\left(L_{+-}\right)$. Then we have

$$
\begin{aligned}
\left\|\left(I-P_{1}\right) x\right\|^{2} & =\|x\|^{2}-\left\|P_{1} x\right\|^{2}=\left\|\Phi_{+-} x\right\|^{2}-\left\|\Phi_{--} P_{1} x\right\|^{2} \\
& =\left\|\Phi_{+-} x\right\|^{2}-\left\|Q_{1}^{-} \Phi_{+-} x\right\|^{2} \\
& =\left(\Phi_{+-} x, \Phi_{+-} x\right)-\left(Q_{1}^{-} \Phi_{+} x, Q_{1}^{-} \Phi_{+-} x\right) \\
& =\int\left(\left(I_{L_{+}-}-Q_{1}^{-}\left(z_{1}\right)^{*} Q_{1}^{-}\left(z_{1}\right)\right)\left(\Phi_{+-} x\right)\left(z_{1}, z_{2}\right),\right. \\
& \left.\left(\Phi_{+-} x\right)\left(z_{1}, z_{2}\right)\right) d m\left(z_{1}, z_{2}\right) .
\end{aligned}
$$

It follows, by putting $x=\left(\Phi_{+-}\right)^{-1} \chi_{E}$ where $\chi_{E}$ is the charactersitic function of the measurable set $E$, that for almost all $\left(z_{1}, z_{2}\right)$, we have $\left(I_{L_{+}-}-Q_{1}^{-}\left(z_{1}\right)^{*} Q_{1}^{-}\left(z_{1}\right)\right) \geqslant 0$. Consequently we can define $\Delta_{1}(z)$ $=\left(I_{L_{+-}}-Q_{1}^{-}\left(z_{1}\right)^{*} Q_{1}^{-}\left(z_{1}\right)\right)^{1 / 2}$ and operator $\Delta_{1}: L^{2}\left(L_{+-}\right) \rightarrow L^{2}\left(L_{+-}\right)$such that $\left(\Delta_{1} f\right)\left(z_{1}, z_{2}\right)=\Delta_{1}\left(z_{1}\right) f\left(z_{1}, z_{2}\right)$ for every $f \in L^{2}\left(L_{+-}\right)$.

Now we have that for $x \in M\left(L_{+_{-}}\right), \quad\left\|\left(I-P_{1} x\right)\right\|=\left\|\Delta_{1} \Phi_{+_{-}} x\right\|$. Consequently we can define a unitary map $\Phi_{u s}$ of $\left(I-P_{1}\right) M\left(L_{+-}\right)$onto $\overline{\Delta_{1} L^{2}\left(L_{+-}\right)}$such that $\Phi_{u s}\left(I-P_{1}\right) x=\Delta_{1} \Phi_{+-} x$ for every $x \in M\left(L_{+-}\right)$. Analogically we can define $\Delta_{2}(z)=\left(I_{L_{-}+}-Q_{2}^{+}(z)^{*} Q_{2}^{+}(z)\right)^{1 / 2}$ for almost every $z \in \Gamma$, the operator $\Delta_{2}: L^{2}\left(L_{-+}\right) \rightarrow L^{2}\left(L_{-+}\right)$such that $\left(\Delta_{2} f\right)\left(z_{1}, z_{2}\right)$ $=\Delta_{2}\left(z_{2}\right) f\left(z_{1}, z_{2}\right)$ for every $f \in L^{2}\left(L_{-+}\right)$and the unitary map $\Phi_{s u}$ : $\left(I-P_{2}\right) M\left(L_{-}\right)$onto $\overline{\Delta_{2} L^{2}\left(L_{-+}\right)}$such that $\Phi_{s u}\left(I-P_{2}\right) x=\Delta_{2} \Phi_{-+} x$ for 
every $x \in M\left(L_{-+}\right)$. Suppose now that $x \in M\left(L_{++}\right)$. Then we have

$$
\begin{aligned}
&\left\|\left(I-P_{1}\right)\left(I-P_{2}\right) x\right\|^{2} \\
&=\|x\|^{2}-\left\|P_{1} x\right\|^{2}-\left\|P_{2} x\right\|^{2}+\left\|P_{1} P_{2} x\right\|^{2} \\
&=\left\|\Phi_{+}+x\right\|^{2}-\left\|\Phi_{-+} P_{1} x\right\|^{2}-\left\|\Phi_{+-} P_{2} x\right\|^{2}+\left\|\Phi_{-} P_{1} P_{2} x\right\|^{2} \\
&=\left\|\Phi_{++} x\right\|^{2}-\left\|Q_{1}^{+} \Phi_{++} x\right\|^{2}-\left\|Q_{2}^{+} \Phi_{++} x\right\|^{2}+\left\|Q_{1}^{-} Q_{2}^{+} \Phi_{++} x\right\|^{2} \\
&= \int_{r^{2}}\left(\left(I_{L}-Q_{1}^{+}\left(z_{1}\right)^{*} Q_{1}^{+}\left(z_{1}\right)-Q_{2}^{+}\left(z_{2}\right)^{*} Q_{2}^{+}\left(z_{2}\right)+\right.\right. \\
&\left.+Q_{2}^{+}\left(z_{2}\right)^{*} Q_{2}^{-}\left(z_{2}\right)^{*} Q_{1}^{-}\left(z_{1}\right) Q_{2}^{+}\left(z_{2}\right)\right)\left(\Phi_{++} x\right)\left(z_{1}, z_{2}\right), \\
&\left.\quad\left(\Phi_{++} x\right)\left(z_{1}, z_{2}\right)\right) d m\left(z_{1}, z_{2}\right) .
\end{aligned}
$$

It follows, by putting $x=\left(\Phi_{++}\right)^{-1} \chi_{E}$ that we can define an operator function

$$
\begin{aligned}
\Delta\left(z_{1}, z_{2}\right)=\left(I_{L}-Q_{1}^{+}\left(z_{1}\right)^{*} Q_{1}^{+}\left(z_{1}\right)-\right. & Q_{2}^{+}\left(z_{2}\right)^{*} Q_{2}^{+}\left(z_{2}\right)+ \\
& \left.+Q_{2}^{+}\left(z_{2}\right)^{*} Q_{1}^{-}\left(z_{1}\right)^{*} Q_{2}^{-}\left(z_{1}\right) Q_{2}^{+}\left(z_{2}\right)\right)^{1 / 2}
\end{aligned}
$$

and an operator $\Delta: L^{2}\left(L_{++}\right) \rightarrow L^{2}\left(L_{++}\right)$such that $(\Delta f)\left(z_{1}, z_{2}\right)=$ $\Delta\left(z_{1}, z_{2}\right) f\left(z_{1}, z_{2}\right)$ for every $f \in L^{2}\left(L_{++}\right)$. Now, for $x \in M\left(L_{++}\right)$we have $\left\|\left(I-P_{1}\right)\left(I-P_{2}\right) x\right\|=\left\|\Delta \Phi_{+} x\right\|$, hence we can define the unitary map $\Phi_{u u}$ of $\left(I-P_{1}\right)\left(I-P_{2}\right) M\left(L_{++}\right)$onto $\overline{\Delta L^{2}\left(L_{++}\right)}$such that $\Phi_{u v}\left(I-P_{1}\right)\left(I-P_{2}\right) x$ $=\Delta \Phi_{++} x$ for every $x \in M\left(L_{++}\right)$.

Let $\Phi=\Phi_{--} \oplus \Phi_{u s} \oplus \Phi_{s u} \oplus \Phi_{u u}$. It is easy to see, by (5.10), that $\Phi$ is unitary map of $K$ onto $L^{2}\left(L_{--}\right) \oplus \overline{\Delta_{1} L^{2}\left(L_{+-}\right)} \oplus \overline{\Delta_{2} L^{2}\left(L_{-+}\right)} \oplus \overline{\Delta L^{2}\left(L_{++}\right)}$. Since there is a unitary equivalence between the spaces $H^{2}\left(L_{--}\right)=$ $\left\{f: D^{2} x \rightarrow L_{-}\right.$such that $f$ is analytic and sup $\int_{r^{2}}\left\|f\left(r_{1} z_{1}, r_{2} z_{2}\right)\right\|^{2} d m\left(z_{1} z_{2}\right)$ $\left.<\infty, 0+r_{1}, r_{2}<1\right\}$ and

$\left\{f \in L^{2}\left(L_{-}\right)\right.$such that $x_{n m}=0$ if $n<0$ or

$$
m<0 \text { where } x_{n m} \text { are Fourier coefficients of } f \text {; }
$$

we can say that $H^{2}\left(L_{--}\right)$is a subspace of $L^{2}\left(L_{--}\right)$. Analogically we can treat the space

$$
\begin{array}{r}
L^{2} \otimes H^{2}(L)=\left\{f: \Gamma \times D \rightarrow L \text { such that, for almost all } z_{1} \in \Gamma, f\left(z_{1}, \cdot\right) \in H_{1}^{2}(L)\right. \\
\text { and for all } \left.z_{2} \in D, f\left(\cdot, z_{2}\right) \in L_{1}^{2}(L)\right\}
\end{array}
$$

as the subspace of $L^{2}(L)$ and $H^{2} \otimes L^{2}(L)$ as the subspace of $L^{2}(L)$. Now it is easy to see by (5.12) that $\Phi K^{+}=H^{2}\left(L_{--}\right) \oplus \Delta_{1} L^{2} \otimes H^{2}\left(L_{+-}\right) \oplus \Delta_{2} H^{2} \otimes$ $\otimes L^{2}\left(L_{-+}\right) \oplus \Delta L^{2}\left(L_{++}\right)$. Consequently, by (5.13) we get that

$$
\Phi H=H^{2}\left(L_{--}\right) \oplus \Delta_{1} L^{2} \otimes H^{2}\left(L_{+--}\right) \oplus \Delta_{2} H^{2} \otimes L^{2}\left(L_{-+}\right) \oplus \Delta L^{2}\left(L_{++}\right) \ominus
$$




$$
\begin{aligned}
\Theta\left\{x \text { where } x \in M^{+}\left(L_{-+}\right) \vee M^{+}\left(L_{+-}\right) \overline{\left(I-P_{1}\right) M_{1} M_{2}^{+}\left(L_{++}\right)} \vee\right. \\
\vee \overline{\left(I-P_{2}\right) M_{1}^{+} M_{2}\left(L_{++}\right)} .
\end{aligned}
$$

Suppose now that $x$ has the form $x=x_{1} \oplus x_{2} \oplus\left(I-P_{1}\right) x_{3}+\left(I-P_{2}\right) x_{4}$ where $x_{1} \in M^{+}\left(L_{+-}\right), \quad x_{2} \in M^{+}\left(L_{-+}\right), \quad x_{3} \in M_{1} M_{2}^{+}\left(L_{++}\right)$. and $x_{4} \in M_{1}^{+} M_{2}\left(L_{++}\right)$. Then

$$
\begin{aligned}
x= & P_{1} P_{2} x_{1} \oplus P_{1} P_{2} x_{2} \oplus P_{1}\left(I-P_{2}\right) x_{1}+P_{1}\left(I-P_{2}\right) x_{3}+P_{2}\left(I-P_{1}\right) x_{2}+ \\
& +P_{2}\left(I-P_{1}\right) x_{4}+\left(I-P_{1}\right)\left(I-P_{2}\right) x_{3}+\left(I-P_{1}\right)\left(I-P_{2}\right) x_{4} \\
= & P_{1} x_{1}+P_{2} x_{2}+\left(I-P_{1}\right) x_{1}+P_{2}\left(I-P_{1}\right) x_{3}+\left(I-P_{2}\right) x_{2}+P_{1}\left(I-P_{2}\right) x_{4}+ \\
& +\left(I-P_{1}\right)\left(I-P_{2}\right) x_{3}+\left(I-P_{1}\right)\left(I-P_{2}\right) x_{4} .
\end{aligned}
$$

Consequently

$$
\begin{aligned}
\Phi x= & \Phi_{--} P_{1} x_{1}+\Phi_{--} P_{2} x_{2}+\Phi_{u s}\left(I-P_{1}\right) x_{1}+ \\
& +\Phi_{u s}\left(I-P_{1}\right) P_{2} x_{3}+\Phi_{s u}\left(I-P_{2}\right) x_{2}+\Phi_{s u}\left(I-P_{2}\right) P_{1} x_{4}+ \\
& +\Phi_{u u}\left(I-P_{1}\right)\left(I-P_{2}\right)\left(x_{3}+x_{4}\right) \\
= & Q_{1}^{-} \Phi_{+-} x_{1}+Q_{2}^{-} \Phi_{-+} x_{2}+\Delta_{1} \Phi_{++} x_{2}+\Delta_{1} Q_{2}^{+} \Phi_{++} x_{3}+ \\
& +\Delta_{2} \Phi_{-+} x_{2}+\Delta_{2} Q_{1}^{+} \Phi_{++} x+\Delta \Phi_{++}\left(x_{3}+x_{4}\right)
\end{aligned}
$$

Now it is easy to see that $\Phi_{x}$, where $x \in M^{+}\left(L_{+-}\right) \vee M^{+}\left(L_{-+}\right)$, $\left(M-P_{1}\right) M_{1} M_{2}^{+}\left(L_{++}\right) \vee\left(I-P_{2}\right) M_{1}^{+} M_{2}\left(L_{++}\right)$has the form

$$
\boldsymbol{\Phi} x=\left(Q_{1}^{-} f_{1}+Q_{2}^{-} f_{2}\right) \oplus\left(\Delta_{1} f_{1}+\Delta_{1} Q_{2}^{+} f_{3}\right) \oplus\left(\Delta_{2} f_{2}+\Delta_{2} Q_{1}^{+} f_{4}\right) \oplus \Delta\left(f_{3}+f_{4}\right)
$$

where $f_{1} \in H^{2}\left(L_{+-}\right), f_{2} \in H^{2}\left(L_{-+}\right), f_{3} \in L^{2} H^{2}\left(L_{++}\right)$and $f_{4} \in H^{2} L^{2}\left(L_{++}\right)$. Let $V_{i}=\left.U_{i}\right|_{K^{+}}$. It is easy to see that $K^{+} \ominus H$ is invariant for $V_{1}$ and $V_{2}$. Consequently, $H$ is invariant for $V_{1}^{*}$ and $V_{2}^{*}$. Since $\left.U_{1}\right|_{H_{.0}}$ and $\left.U_{2}\right|_{H_{0}}$ are minimal unitary dilations of $T_{1}$ and $T_{2}$ respectively, $\left.V_{1}\right|_{H_{.0}^{+}} ^{+}=\left.U_{1}\right|_{H_{.0}^{+}}$and $\left.V_{1}\right|_{H_{0}^{+}}=\left.U_{1}\right|_{H_{0}^{+}}$are minimal isometric dilations of $T_{1}$ and $T_{2}$ respectively. Since $H_{.0}^{+}$reduces $V_{1}$ and $H_{0}^{+}$reduces $V_{2}$ we have $T_{1}^{*} x$ $=\left(\left.V_{1}\right|_{H_{0}^{+}}\right)^{*} x=V_{1}^{*} x$ and $T_{2}^{*}=\left(\left.V_{2}\right|_{H_{0}^{+}}\right)^{*} x=V_{2}^{*} x$ for every $x \in H$. So we have to find $\Phi V_{1}^{*} \Phi^{-1}$ and $\Phi V_{2}^{*} \Phi^{-1}$. First we shall show that $M^{+}\left(L_{--}\right)$, $\left(I-P_{1}\right) M_{1} M_{2}^{+}\left(L_{+-}\right), \quad\left(I-P_{2}\right) M_{1}^{+} M_{2}\left(L_{-+}\right)$and $\left(I-P_{1}\right)\left(I-P_{2}\right) M\left(L_{++}\right)$ reduce $V_{1}$ and $V_{2}$. Since these spaces span $K^{+}$(see (5.12)) it is sufficient to show that they are invariant for $V_{1}$ and $V_{2}$. It is easy to see that $M^{+}\left(L_{--}\right)$is invariant for $U_{1}$ and $\left.U_{2}\right|_{M+}{ }_{\left(L_{-}\right)}=\left.V_{1}\right|_{M^{+}\left(L_{--}\right)}$is a unilateral shift. Since the space $M_{1} M_{2}^{+}\left(L_{+-}\right)$reduces $\bar{U}_{1}^{-}$by the commutativity of $U_{1}$ and $P_{1}$ we get that $\left(I-P_{1}\right) M_{1} M_{2}^{+}\left(L_{+-}\right)$reduces $U_{1}$. Consequently $V_{1} l_{\left(I-P_{1}\right) M_{1} M_{2}^{+}\left(L_{+}\right)}$ $=\left.U_{1}\right|_{\left(I-P_{1}\right) M_{1} M_{2}^{+}\left(L_{+} \vdots\right)}$. By commutativity of $U_{1}$ and $P_{2}$ we get that $\left(I-P_{2}\right) M_{1}^{+} M_{2}\left(L_{-}+\right)$is an invariant subspace for $U_{1}$ and consequently $\left.V_{1}\right|_{\left(I-P_{2}\right) M_{1}^{+} M_{2}\left(L_{-+}\right)}$is a unilateral shift. Since $M\left(L_{++}\right)$reduce $U_{1}$ we may 
conclude that $\left.V_{1}\right|_{\left(I-P_{1}\right)\left(I-P_{2}\right) M\left(L_{+}\right)}=\left.U_{1}\right|_{\left(I-P_{1}\right)\left(I-P_{2}\right) M\left(L_{+}\right)}$. It is easy to see that for $\left.V_{1}\right|_{M^{+}\left(L_{-}\right)}$, the space $M_{2}^{+}\left(L_{-}\right)$is the complete wandering subspace (a wandering subspace $L$ for the isometry $V$ on $H$ is called complete if $\left.H=\bigoplus_{n=0}^{\infty} V^{n} L\right)$. Consequently, $V_{1}^{*}\left(\sum_{n=0}^{\infty} U_{1}^{n} x_{n}\right)=U_{1}^{*}\left(\sum_{n=0}^{\infty} U_{1}^{n} x_{n}-x_{0}\right)$ where $x_{n} \in M_{2}^{+}\left(L_{--}\right)$. Similarly if $x_{n} \in M_{2}\left(L_{-+}\right)$then

$$
V_{1}^{*}\left(I-P_{1}\right)\left(\sum_{n=-\infty}^{\infty} U_{1}^{n} x_{n}\right)=U_{1}^{*}\left(I-P_{1}\right)\left(\sum_{n=-\infty}^{\infty} U_{1}^{n} x_{n}-x_{0}\right)
$$

If $x=\sum_{n, m=-\infty}^{\infty} U_{1}^{n} U_{2}^{m} x_{n m}$ where $x_{n m} \in L_{--}$and $f=\Phi_{--} x$ then $f\left(z_{1}, z_{2}\right)$ $=\sum_{n, m=-\infty}^{\infty} z_{1}^{n} z_{2}^{m} x_{n m}$. It follows that

$$
\begin{array}{r}
\left(\Phi U_{1}^{*} x\right)\left(z_{1}, z_{2}\right)=\left(\Phi--U_{1}^{*} x\right)\left(z_{1}, z_{2}\right)=\Phi\left(\sum_{n, m=-\infty}^{\infty} U_{1}^{n-1} U_{2}^{m} x_{n m}\right)\left(z_{1}, z_{2}\right) \\
=\sum_{n, m=-\infty}^{\infty} z_{1}^{n-1} z_{2}^{m} x_{n m}=1 / z_{1}\left(\sum_{n, m=-\infty}^{\infty} z_{1}^{n} z_{2}^{m} x_{n m}\right)=1 / z_{1} f\left(z_{1}, z_{2}\right) .
\end{array}
$$

Evidently $\Phi\left(\sum_{n=-\infty}^{\infty} U_{2}^{m} x_{0 m}\right)=\sum_{m=-\infty}^{\infty} z_{2}^{m} x_{0 m}=f\left(0, z_{2}\right)$. Consequently, we have that $\left(\Phi V_{1}^{*} \Phi^{*} f\right)\left(z_{1}, z_{2}\right)=1 / z_{1}\left(f\left(z_{1}, z_{2}\right)-f\left(0, z_{2}\right)\right)$ for every $f \in H^{2}\left(L_{--}\right)$. A similar proof shows that $\Phi V_{1}^{*} \Phi^{-1}$ has the same form for $f \in H^{2} L^{2}\left(L_{-+}\right)$. It is easy to see that if $\Delta_{1} L^{2} H^{2}\left(L_{+-}\right)$or if $f \in L^{2}\left(L_{++}\right)$then $\left(\Phi V_{1} \Phi^{-1}\right)(f)\left(z_{1}, z_{2}\right)=1 / z_{1}(f)\left(z_{1}, z_{2}\right)$. Analogically we prove that for $f \in H^{2}\left(L_{--}\right)$or $f \in \Delta_{1} L^{2} H^{2}\left(L_{+-}\right)\left(\Phi V_{2}^{*} \Phi^{-1}\right) f\left(z_{1}, z_{2}\right)=1 / z_{2}\left(f\left(z_{1}, z_{2}\right)-\right.$ $\left.-f\left(z_{1}, 0\right)\right)$ and for $f \in \Delta_{2} H^{2} L\left(L_{-+}\right)$or $f \in \Delta L^{2}\left(L_{++}\right),\left(\Phi V_{2} \Phi^{-1}\right)(f)\left(z_{1}, z_{2}\right)$ $=1 / z_{2} f\left(z_{1}, z_{2}\right)$. Since at the beginning we could have put $T_{1}^{*}$ and $T_{2}^{*}$ in place of $T_{1}$ and $T_{2}$, we have proved the following

THEOREM 1. Suppose that $T_{1}$ and $T_{2}$ are completely nonunitary doubly commuting contractions on the separable Hilbert space $H$. Let $U_{1}, U_{2}$ be the minimal unitary dilation of the pair $T_{1}, T_{2}$, as in Theorem 0 . Then there are bounded analytic operator functions $\left\{Q_{1}^{+}, L_{++}, L_{-+}\right\},\left\{Q_{1}^{-}, L_{+-}, L_{--}\right\}$, $\left\{Q_{2}^{+}, L_{++}, L_{+-}\right\}$and $\left\{Q_{2}^{-}, L_{-+}, L_{--}\right\}$such that the space $H$ is unitarily equivalent to the space

$$
\begin{aligned}
& H=H^{2}\left(L_{--}\right) \oplus \Delta_{1} L^{2} H^{2}\left(L_{+_{-}}\right) \oplus \Delta_{2} H^{2} L^{2}\left(L_{-+}\right) \oplus \Delta L^{2}\left(L_{++}\right) \Theta \\
& \Theta\left\{\left(Q_{1}^{-} f_{1}+Q_{2}^{-} f_{2}\right) \oplus\left(\Delta_{1} f_{1}+\Delta_{1} Q_{2}^{+} f_{3}\right) \oplus\left(\Delta_{2} f_{2}+\Delta_{2} Q_{1}^{+} f_{4}\right) \oplus \Delta\left(f_{3}+f_{4}\right)\right.
\end{aligned}
$$$$
\text { where } \left.f_{1} \in H^{2}\left(L_{+-}\right), f_{2} \in H^{3}\left(L_{-+}\right), f_{3} \in L^{2} H^{2}\left(L_{++}\right) \text {and } f_{4} \in H^{2} L^{2}\left(L_{++}\right)\right\}
$$ 
where

$$
\begin{gathered}
L_{--}=\overline{\left(I-U_{1} T_{1}^{*}-U_{2} T_{2}^{*}+U_{1} U_{2} T_{1}^{*} T_{2}^{*}\right) H}, \\
L_{+-}=\overline{\left(U_{1}-T_{1}-U_{1} U_{2} T_{2}^{*}+U_{2} T_{1} T_{2}^{*}\right) H}, \\
L_{-+}=\overline{\left(U_{2}-T_{2}-U_{1} U_{2} T_{1}^{*}+U_{1} T_{1}^{*} T_{2}\right) H} \\
L_{++}=\overline{\left(U_{1} U_{2}-U_{2} T_{2}-U_{2} T_{1}+T_{1} T_{2}\right) H}, \\
\left(Q_{i}^{a} f\right)\left(z_{1}, z_{2}\right)=Q_{i}^{a}\left(z_{i}\right) f\left(z_{1}, z_{2}\right) \quad(i=1,2, a=+,-), \\
\Delta_{i} f\left(z_{1}, z_{2}\right)=\Delta_{i}\left(z_{i}\right) f\left(z_{1}, z_{2}\right), \quad \Delta_{i}(z)=\left(I-Q_{i}^{+}(z)^{*} Q_{i}^{+}(z)\right)^{1 / 2} \quad(i=1,2), \\
\quad(\Delta f)\left(z_{1}, z_{2}\right)=\Delta\left(z_{1}, z_{2}\right) f\left(z_{1}, z_{2}\right), \\
\Delta\left(z_{1}, z_{2}\right)=\left(I-Q_{1}^{+}\left(z_{1}\right)^{*} Q_{1}^{+}\left(z_{1}\right)-Q_{2}^{+}\left(z_{2}\right)^{*} Q_{2}^{+}\left(z_{2}\right)+\right. \\
\left.\quad+Q_{2}^{+}\left(z_{2}\right)^{*} Q_{1}^{-}\left(z_{2}\right) Q_{1}^{-}\left(z_{1}\right) Q_{2}^{+}\left(z_{2}\right)\right)^{1 / 2} .
\end{gathered}
$$

Operators $T_{1}$ and $T_{2}$ are unitarily equivalent to the operators $\tilde{T}_{1}$ and $\tilde{T}_{2}$ on $H$ such that for $f=f_{1}+f_{2}+f_{2}+f_{4}$ where

$$
\begin{aligned}
& f_{1} \in H^{2}\left(L_{-}\right), \quad f_{2} \in \overline{\Delta_{1} L^{2} H^{2}\left(L_{+-}\right)}, \quad f_{3} \in \overline{\Delta_{2} \overline{H^{2} L^{2}\left(L_{-+}\right)}}, \quad f_{4} \in \overline{\Delta L^{2}\left(L_{++}\right)}, \\
& \left(\tilde{T}_{1} f_{1}\right)\left(z_{1}, z_{2}\right)=1 / z_{1}\left(f_{1}\left(z_{1}, z_{2}\right)-f_{1}\left(0, z_{2}\right)\right), \quad\left(\tilde{T}_{1} f_{2}\right)\left(z_{1}, z_{2}\right)=1 / z_{1}\left(f_{2}\left(z_{1}, z_{2}\right)\right), \\
& \left(\tilde{T}_{1} f_{3}\right)\left(z_{1}, z_{2}\right)=1 / z_{1}\left(f_{3}\left(z_{1}, z_{2}\right)-f_{3}\left(0, z_{2}\right)\right), \quad\left(\tilde{T}_{1} f_{4}\right)\left(z_{1}, z_{2}\right)=1 / z_{1}\left(f_{4}\left(z_{1}, z_{2}\right)\right), \\
& \left(\tilde{T}_{2} f_{1}\right)\left(z_{1}, z_{2}\right)=1 / z_{2}\left(f_{1}\left(z_{1}, z_{2}\right)-f_{1}\left(z_{1}, 0\right)\right), \quad\left(\tilde{T}_{2} f_{3}\right)\left(z_{1}, z_{2}\right)=1 / z_{2}\left(f_{3}\left(z_{1}, z_{2}\right)\right), \\
& \left(\tilde{T}_{2} f_{2}\right)\left(z_{1}, z_{2}\right)=1 / z_{2}\left(f_{2}\left(z_{1}, z_{2}\right)-f_{2}\left(z_{1}, 0\right)\right), \quad\left(\tilde{T}_{2} f_{4}\right)\left(z_{1}, z_{2}\right)=1 / z_{2}\left(f_{4}\left(z_{1}, z_{2}\right)\right) \text {. }
\end{aligned}
$$

Now we consider the case where $T_{1}$ is completely nonunitary and $T_{2}$ is unitary. If $U_{1}$ is the minimal unitary dilation of $T_{1}$ then there is exactly one extension $U_{2}$ of $T_{2}$ such $U_{2}$ commutes with $U_{1}$ and $U_{2}$ is a extension of $T_{2}$. Moreover, $U_{2}$ is unitary and the pair $U_{1}, U_{2}$ is the minimal unitary dilation of the pair $T_{1}, T_{2}$. By Theorem VI.2.1 there is a bounded analytic function $Q$, $L_{T_{1}}^{+}, U, L_{T_{1}}^{-}$such that $H$ is unitarily equivalent to the space

$$
H=H_{1}^{2}\left(L_{T_{1}}^{-}\right) \oplus \Delta L_{1}^{2}\left(L_{T_{1}}^{+}\right) \Theta\left\{Q f+\Delta f \text { where } f \in H_{1}^{2}\left(L_{T_{2}}^{+}\right)\right\}
$$

where $\Delta(z)=\left(I-(Q)(z)^{*} Q(z)\right)^{1 / 2}$ and $T_{1}$ is unitarily equivalent to the operator $T_{1}$ on such that for $f=f_{1}+f_{2}$, where $f_{1} \in H_{1}^{2}\left(U_{1} L_{T_{1}}^{-}\right)$and $f_{2} \in L_{1}^{2}\left(L_{T_{1}}^{+}\right)$, $\left(T_{1} f_{1}\right)(z)=1 / z\left(f_{1}(z)-f_{1}(0)\right)$ and $\left(T_{1} f_{2}\right)(z)=1 / z\left(f_{2}(z)\right)$. Now if we apply Lemma V.3.2 to the operators $\left.U\right|_{M_{1}^{+}\left(U_{1} L_{T_{1}}^{-}\right)}$and $\left.U\right|_{M_{1}^{+}\left(L_{T_{1}}^{-}\right)}$we get the following:

THEOREM 2. Suppose that $T_{1}$ is completely nonunitary contraction on $H$. Let $U$ be unitary operator on $H$ which commutes with $T_{1}$. If $U_{1}$ is the minimal unitary dilation of $T_{1}$, then there is the analytic bounded operator function 
$\left\{Q, L_{+}, L_{+}\right\}$such that $H$ is unitarily equivalent to the space

$$
H=\left(H_{1}^{2}\left(L_{-}\right) \oplus L_{1}^{2}\left(L_{+}\right)\right) \ominus\left\{Q f+\Delta f \text { where } f \in H_{1}^{2}\left(L_{+}\right)\right\}
$$

where $L_{-}=\overline{\left(I-U_{1} T_{1}^{*}\right) H}, \quad L_{+}=\overline{\left(U_{1}-T_{1}\right) H}, \quad \Delta(z)=\left(I-Q(z)^{*} Q(z)\right)^{1 / 2}$. Operators $T_{1}$ and $U$ are unitarily equivalent to the operators $\tilde{T}_{1}$ and $\tilde{U}$, such that for $f=f_{1}+f_{2}$ where $f_{1} \in H_{1}\left(L_{-}\right)$and $f_{2} \in \Delta L_{1}\left(L_{+}\right)$we have

$$
\begin{array}{cc}
\left(\tilde{T}_{1} f_{1}\right)(z)=1 / z\left(f_{1}(z)-f_{1}(0)\right), & \left(\tilde{T}_{1} f_{2}\right)(z)=1 / z\left(f_{2}(z)\right), \\
\tilde{U} f_{1}(z)=U_{-} f_{1}(z) \quad \text { and } & \left(\tilde{U} f_{2}\right)(z)=U_{+} f_{2}(z)
\end{array}
$$

where, for $x \in H$,

$$
U_{-}\left(I-U_{1} T_{1}\right) x=\left(I-U_{1} T_{1}\right) U x \text { and } U_{+}\left(U_{1}-T_{1}\right) x=\left(U_{1}-T_{1}\right) U x .
$$

\section{References}

[1] Sz. Nagu, B. Foias,, Analyse harmonique des operateurs de respace de Hilbert, Masson et C Academial Kiado, 1967.

[2] M. Slociński, On an isometric dilations of commuting contractions and related model (in preparation).

POLISH ACADEMY OF SCIENCES, SECTION CRACOW 